This item was submitted to Loughborough's Research Repository by the author.

Items in Figshare are protected by copyright, with all rights reserved, unless otherwise indicated.

\title{
Passive scalar diffusion in three-dimensional turbulent rectangular free jets with numerical evaluation of turbulent Prandtl/Schmidt number
}

PLEASE CITE THE PUBLISHED VERSION

https://doi.org/10.1016/j.icheatmasstransfer.2018.03.019

\section{PUBLISHER}

(C) Elsevier

VERSION

AM (Accepted Manuscript)

\section{PUBLISHER STATEMENT}

This work is made available according to the conditions of the Creative Commons Attribution-NonCommercialNoDerivatives 4.0 International (CC BY-NC-ND 4.0) licence. Full details of this licence are available at: https://creativecommons.org/licenses/by-nc-nd/4.0/

\section{LICENCE}

CC BY-NC-ND 4.0

\section{REPOSITORY RECORD}

Di Venuta, Ivan, Andrea Boghi, Matteo Angelino, and Fabio Gori. 2018. "Passive Scalar Diffusion in Threedimensional Turbulent Rectangular Free Jets with Numerical Evaluation of Turbulent Prandt//schmidt Number". figshare. https://hdl.handle.net/2134/32018. 


\title{
Passive Scalar Diffusion in Three-Dimensional Turbulent Rectangular Free Jets with numerical evaluation of turbulent Prandtl/Schmidt number
}

\author{
Ivan Di Venuta ${ }^{\mathrm{a}}$, Andrea Boghi ${ }^{\mathrm{b}}$, Matteo Angelino ${ }^{\mathrm{c}}$, Fabio Gori ${ }^{\mathrm{a}, *}$ \\ ${ }^{a}$ Department of Industrial Engineering, University of Rome “Tor Vergata”, Via del Politecnico 1, 00133 Rome, Italy. \\ bSchool of Water, Energy and Environment, Cranfield University, Cranfield, Bedordshire, MK43 0AL, UK. \\ ${ }^{c}$ Loughborough University, Aeronautical and Automotive Engineering, Stewart Miller Building, Loughborough, LE11 3TU, UK. \\ ${ }^{*}$ Corresponding Author, gori@uniroma2.it
}

\begin{abstract}
The passive scalar spreading of fluids with laminar Prandtl or Schmidt number, $\operatorname{Pr}, S_{c}$, equal to 1 in turbulent rectangular submerged free jets is analyzed by means of numerical simulation and theoretical analysis in the Reynolds number range 5000-40,000. The numerical investigation is carried out by means of a three-dimensional (3D) Large Eddy Simulation (LES) approach with the dynamic Smagorinsky model. A new mathematical model allows to obtain a simplified description of the passive scalar spreading in the largest area of the flow field, the Fully Developed Region (FDR). The present three-dimensional (3D) investigation shows that the passive scalar spreading follows a self-similarity law in the Fully Developed Region (FDR), as well as in the mean Undisturbed Region of Flow (URF) and in the Potential Core Region (PCR), similarly to what found in the Near Field Region (NFR) of rectangular submerged free jets, investigated with a two-dimensional (2D) approach. The turbulent Prandtl or Schmidt number is evaluated numerically and is found to be inversely proportional to the mean velocity gradient in the PCR. The present 3D numerical results show that the turbulent Prandtl or Schmidt number is zero in most part of the mean URF, and PCR, while it assumes different values outside. In the FDR the turbulent Prandtl or Schmidt number is constant and approximately equal to 0.7 , in agreement with the literature, showing that turbulence affects momentum and passive scalar in a different way.
\end{abstract}

Keywords: Submerged rectangular free jet of fluids with $\mathrm{Pr} / \mathrm{Sc}$ equal to one; $3 \mathrm{D}$ turbulent flow and passive scalar; Turbulent Prandtl/Schmidt number evaluation; Large eddy simulation; Self-Similarity of the fully developed region.

\section{Nomenclature}

D diameter

$f \quad$ instantaneous self-similarity function

$U \quad$ mean axial velocity

$h \quad$ half-height of the slot

$u \quad$ instantaneous axial velocity

$k \quad$ turbulent kinetic energy

$V \quad$ mean transverse velocity

$L_{s} \quad$ virtual origin of the FDR

$v \quad$ instantaneous transverse velocity

$P \quad$ mean static pressure

$x \quad$ axial coordinate

$p \quad$ instantaneous static pressure

$y \quad$ transverse coordinate

$r \quad$ particle radius

Dimensionless parameters

$S_{i j} \quad$ rate of shear tensor

c Tollmien's coefficient

$g$ Görtler's coefficient

$t$ time

$P e=R e \times S c \quad$ Peclet number 
$R e=\frac{U_{i n} D_{h}}{v} \quad$ Reynolds number

Sc $=\frac{v}{\Gamma} \quad$ Schmidt number

$\operatorname{Pr}_{T}=S c_{T} \quad$ Turbulent Prandtl/ Schmidt

number

$\mathrm{Ti} \quad$ Turbulence intensity

Greek

$\dot{\gamma} \quad$ shear rate

$\Gamma \quad$ Passive scalar molecular diffusivity

$\Delta \quad$ filter width

$\zeta \quad$ Tollmien's self-similarity variable

$\eta \quad$ self-similarity variable

$\mu \quad$ dynamic viscosity

$v \quad$ kinematic viscosity

$v_{T} \quad$ turbulent viscosity

$\xi \quad$ Görtler’s self-similarity variable

$\tau_{i j}^{R} \quad$ Reynolds stress tensor

$\phi \quad$ instantaneous passive scalar

$\Phi \quad$ mean passive scalar

$\psi \quad$ instantaneous stream-function

$\Psi \quad$ mean stream-function

$\hat{\phi} \quad$ scaled passive scalar

\section{Subscripts}

C centerline

h hydraulic

in inlet

sgs sub-grid scale

T turbulent 


\section{Introduction}

The investigation on the value of the turbulent Prandtl, $P r_{T}$, to be used in the numerical evaluation of turbulent flows, started with the considerations of Reynolds, [1], about the relation between local shearing stress and local heat flux. A series of papers solved theoretically and numerically several different turbulent problems with the so-called Reynolds analogy, $\operatorname{Pr}_{T}=1$, starting from the early 60's [2]. A simple model of turbulent heat/mass transfer, based on a modified form of the Reynolds analogy, was proposed in [3], by deriving equations from which heat/mass transfer coefficients and temperature/concentration profiles may be predicted at any value of the molecular or laminar Prandtl or Schimdt number.

The problem of heat transfer across turbulent, incompressible boundary layers was investigated in [4] with the conclusion that $\operatorname{Pr}_{T}$ can be assumed constant, in the range between 1 and 0.78 . This range of values was extended to $0.5-1.5$ in [5], while it was assumed equal to 0.7 in [6] and 0.85 in [7]. More than thirty models were examined in [8] for the prediction of the relationship between turbulent transfer of momentum and passive contaminant, such as heat or dissolved matter, by dividing them into seven classes, and considering also the flow of liquid metals for which $\operatorname{Pr}_{T}$ can be higher than 1 . The same problem for free flow of air (e.g. wakes and jets) was reviewed in [9], confirming that the value of $\operatorname{Pr}_{T}=S c_{T}$ is around 1. The proposal of a turbulent Prandtl number for liquid metals, done in [10], was then applied to the numerical simulations of laminar and turbulent liquid metals flow, [11-13]. In the calculation of heat, mass and momentum transport in coaxial jets and mixing layers, [14], the value of 0.70 for $P r_{T}=S c_{T}$ was then assumed. The turbulent Prandtl number, $P r_{T}$, was measured in a heated circular jet into still air, indicating that it increases near the edge of the jet but is approximately constant $(0.81 \pm 0.05)$ in a region between the axis and the jet half-radius, [15].

In the mass transfer of turbulent impinging slot jets, the turbulent Schmidt number, $S c_{T}$, was assumed equal to 0.73 and 0.9, at the several Reynolds numbers investigated in [16]. Turbulent mass transfer was studied in [17] obtaining a value of $S c_{T}$ in the range from 0.2 to 1.5, and finding a significant influence in the prediction of the concentration in jet-in-cross flows. As far as jet is concerned, the RANS investigations of [18], which used the realizable $k-\varepsilon$ model for an axisymmetric turbulent round jet showed the influence of $S c_{T}$ on the solution, with the conclusion that $S c_{T}=0.7$ should be recommended for axisymmetric free-jet flows. 
The turbulent $S c_{T}$ was derived numerically in [19] with the DNS approach for a round turbulent jet in the Fully Developed Region (from 20 to 37.5 hydraulic diameters), obtaining an average value of 0.74 in the radial coordinate, in sufficient agreement to the experimental values of [15], which were in the range between 0.65 and 0.81 . The DNS approach was extended to turbulent channel flow at high Schmidt numbers evaluating the turbulent Schmidt number, which was ranging from $S c_{T}=2.2$, for $S c=49$, towards unity, for $S c=3$, close to the wall, down to unity for all the $S c$ numbers, [20].

The experimental flow evolution of a two-dimensional (2D) rectangular jet was described in [21] with the definition of the potential core region (PCR), or zone of flow establishment, where the average velocity on the jet centerline remains equal to the exit one, and the fully developed region (FDR), after the PCR, or zone of established flow. The deformation of a three-dimensional (3D) rectangular jet was investigated in [22], where the line-source of the jet was found independent from the Reynolds number. Tollmien [23] and Görtler [24] studied theoretically the flow of turbulent rectangular submerged free jets and proposed a self-similar evolution of the axial velocity in the PCR and FDR. The equations describing the velocity evolution were confirmed experimentally in [25-27], for the PCR, and in [28], for the FDR.

The experimental mean concentration visualizations and fluid dynamics measurements, carried out in [29-34], showed that velocity and turbulence remain the same of those measured on the exit, for a length of flow called Undisturbed Region of Flow, URF. Experimental instantaneous visualizations and measurements of the flow evolution, [35], evidenced the presence of two types of flow before the vortex breakdown. In the first type of flow the jet height maintains constant and is called Negligible Disturbances Flow (NDF), while, in the second one, the jet height oscillates, with contractions and/or enlargements but without the vortex formation, and is called Small Disturbances Flow (SDF). Numerical investigations have reproduced, with the Reynolds Averaged Navier-Stokes (RANS) equations, the URF, [36-37], and, later on, with the Large Eddy Simulations (LES) approach, the URF, the NDF and the SDF [38-39]. The last two studies showed that the velocity profile in the URF obeys a self-similar law, different from those proposed for the PCR in [23-24].

Fluid dynamics strongly influences heat and mass transfer in free jets and between jet and solid, or liquid surface. The parameters determining the dispersion of particles, or droplets, in jets, [40-42], and the heat transfer from a jet to a solid surface, [43-44], are the Reynolds number (Re) and the turbulence intensity (Ti). In applications like micro-jets for drug injection, [45-46], and recent welding technologies, [47-48], low turbulence intensities and moderate Reynolds numbers are requested, evidencing the role of molecular diffusion. The diffusivity of a particle is proportional to its radius, according to the Stoke-Einstein relation, [49], and the Cunningham empirical equation, [50], giving a Schmidt number of air in the range from 1 to 100 for particle radius of the order of $r \approx 10^{-7}-10^{-5} \mathrm{~m}$. The diffusion of the passive scalar in turbulent planar jets has been investigated in the URF and PCR in [51], but not in the FDR, finding that the turbulent Prandtl 
or Schmidt number, $P r_{T}=S c_{T}$, is inversely proportional to the mean velocity gradient. Comparisons with the mean and instantaneous PIV and HFA measurements were done in [52]. A mathematical formulation of the mean passive scalar diffusion in the FDR, i.e. the "Gaussian Plume Model” (GPM), is limited to circular jets [53-54]. The GPM, widely used in environmental applications [55], was derived from the RANS equations of turbulent flow under the hypotheses of constant eddy diffusivities for both momentum and passive scalar, due to the difficulties in the modeling of the turbulent Prandtl or Schmidt number, $\operatorname{Pr}_{T}, S c_{T}$.

This work presents the results of three-dimensional (3D) Large Eddy Simulations (LES) of rectangular free jets in the Fully Developed Region, FDR. The numerical simulations cover the range of turbulent Reynolds number from 5000 to 40,000, for a molecular Prandtl or Schmidt number, $\mathrm{Pr}$, Sc , equals to 1, since the spreading of the passive scalar in the PCR and FDR is governed only by the eddy diffusivity. This work extends the 2D approach, employed in [51], showing that with the 3D approach it is possible to define a new theory for the diffusion of the passive scalar in the FDR.

Another goal of the paper is to evaluated numerically the turbulent Prandtl or Schmidt number $P r_{T}, S c_{T}$ which is important to be used as input in the RANS modeling for computing the heat transfer in a jet impinging a single smooth cylinder [56-62], a finned cylinder [63-67], two [68, 69] and three cylinders in a row $[70,71]$.

\section{Numerical Method}

\subsection{Governing Equations}

The Large Eddy Simulation (LES) approach allows to solve the large-scale turbulent structure and to model small-scale ones through a spatial filtering of the Navier-Stokes equations, leading to a reduction of the computational costs, but allowing to capture the fluid dynamics. If $a\left(t, x_{i}\right)$ is a generic field, function of the time $t$ and the spatial coordinate, $x_{i}$, a grid-scale filtered field $\tilde{a}\left(t, x_{i}\right)$ can be defined as

$$
\tilde{a}\left(t, x_{i}\right)=\int_{\Omega} a\left(t, \xi_{i}\right) g\left(x_{i}-\xi_{i}, \Delta\right) d^{3} \xi_{i}
$$

where $\Omega$ is the domain extension and $g$ the spatial filter, function of the width $\Delta$.

The application of the filtering approach to the conservation equations, expressed in non-dimensional form, allows obtaining the following equations for the conservation of mass

$$
\frac{\partial \tilde{u}_{i}}{\partial x_{i}}=0
$$

momentum 
$\frac{\partial \tilde{u}_{i}}{\partial t}+\frac{\partial}{\partial x_{j}}\left(\tilde{u}_{i} \tilde{u}_{j}+\tilde{p} \delta_{i j}-\frac{2}{\operatorname{Re}} \tilde{S}_{i j}\right)=\frac{\partial \tau_{i j}^{s g s}}{\partial x_{j}}$

and passive scalar

$\frac{\partial \tilde{\phi}}{\partial t}+\frac{\partial}{\partial x_{j}}\left(\tilde{\phi} \tilde{u}_{j}-\frac{1}{\operatorname{Re} \cdot \operatorname{Sc}} \frac{\partial \tilde{\phi}}{\partial x_{j}}\right)=-\frac{\partial J_{j}^{s g s}}{\partial x_{j}}$

being $S_{i j}$ the rate of shear tensor, $u_{i}$ the velocity vector, $p$ the static pressure, $\delta_{i j}$ the identity tensor, and $\phi$ the passive scalar. The sub-grid stress tensor, $\tau_{i j}^{\text {sgs }}$, is modeled with the diffusive gradient hypothesis $\tau_{i j}^{s g s}=2 v_{s g s} S_{i j}-\frac{2}{3} k_{s g s} \delta_{i j}$

Similarly, the sub-grid mass flux vector, $J_{k}^{s g s}$, is

$$
J_{k}^{s g s}=-\frac{v_{s g s}}{S c_{s g s}} \frac{\partial \tilde{\phi}}{\partial x_{k}}
$$

where $S c_{s g s}$ is the sub-grid Schmidt number. The sub-grid viscosity, $v_{s g s}$ and sub-grid kinetic energy, $k_{s g s}$ are

$v_{s g s}=C_{s} \Delta^{2} \tilde{S}$

$k_{\text {sgs }}=C_{I} \Delta^{2} \tilde{S}^{2}$

where the filtered shear rate, $\tilde{S}$, and the filter width, $\Delta$, are

$\tilde{S}=\sqrt{2 \tilde{S}_{i j} \tilde{S}_{i j}}$

$\Delta=\sqrt{\Delta_{k} \Delta_{k}}$

with $\Delta_{k}$ the box filter width in the k-th direction. The dynamic Smagorinsky model, [72], is employed.

The turbulent viscosity and the turbulent Prandtl or Schmidt number $P r_{T}, S c_{T}$ are evaluated with the present numerical LES approach. The turbulent viscosity, defined as the ratio between the mean shear rate and the second invariant of the Reynolds stress tensor, is isotropic, i.e. is independent on the flow direction, and is given by 
$v_{T}=\frac{1}{\overline{\dot{\gamma}}} \sqrt{\frac{1}{2} \tau_{i j}^{R} \tau_{i j}^{R}-\frac{2}{3} k^{2}}$

where $\overline{\dot{\gamma}}$ is the mean shear rate, $\tau_{i j}^{R}$ the Reynolds stress tensor and $k$ the turbulent kinetic energy. The turbulent Prandtl or Schmidt number, $P r_{T}, S c_{T}$, defined as the ratio between the mean passive scalar gradient and the mean turbulent passive scalar flux, is isotropic, i.e. is independent on the flow direction, and is given by

$P r_{T}=S c_{T}=v_{T} \sqrt{\frac{\partial \Phi}{\partial x_{j}} \frac{\partial \Phi}{\partial x_{j}}}\left(\sqrt{\overline{\phi^{\prime} u_{k}^{\prime}} \phi^{\prime} u_{k}^{\prime}}\right)^{-1}$

\subsection{Computational details}

The simulations are realized with the pisoFoamPS finite-volume solver, implemented in OpenFOAM. The 3D grid is generated with blockMesh, the OpenFOAM utility for mesh generation, at all the Reynolds numbers. The geometry is made of a rectangular box, 2 diameter long in the transverse direction ( $z$ ), 3 diameters in the vertical $(y)$ and 6.5 diameters in the axial direction $(x)$. The grid is uniform in the $z$ direction and stretched in the $x$ and $y$ directions near the slot exit, although the minimum and maximum grid stencils are of the same order of magnitude. In the $z$ direction the stencil is $\Delta z=0.0197$ hydraulic diameters, while in the $x$ and $y$ directions they are $\Delta x, \Delta y=0.0074 \div 0.0294$ hydraulic diameters. The grid is made of $442 \times 262 \times 102$ points, respectively in the $x, y$ and $z$ directions. The explicit time integration scheme is the second order backwards, and is second order central for the spatial derivatives. The time step in each simulation satisfies the condition CFL $<0.5$. The filter chosen in OpenFOAM is the simple one and the filter amplitude is the cubic root of the cell volume.

A wall is present above and below the slot, with a thickness of 0.176 hydraulic diameters, equal to the experimental conditions [34,35]. Everywhere else, i.e. in the $x$ and $y$ direction, a free boundary is present. The total pressure is imposed on the outlet boundaries, as well as the velocity boundary condition, which changes according to its direction. If the fluid flows out of the domain a zero gradient condition is imposed for the velocity, otherwise the normal velocity gradient is obtained from the internal-cell value. This boundary condition in OpenFOAM is known as "pressureInletOutletVelocity". A periodic boundary condition is imposed in the streamwise direction.

A top-hat velocity profile is assumed on the slot exit, with the addition of a small perturbation of $1 \%$ in the amplitude, which triggers the 3D flow, even with 2D boundary conditions, as shown in [39]. The simulations are carried on for 30 flow times, being the flow time the ratio between the domain length and the axial velocity on the slot exit. The steady state is reached after 10 flow times and the following 20 flow times are employed to obtain the mean fields. 


\section{Numerical results}

\subsection{Instantaneous fields}

Figure 1 reports the instantaneous passive scalar fields for Reynolds number equal to 5000, 10,000, 20,000, 40,000. In the instantaneous flow evolution the Negligible Disturbances Flow, NDF, and the Small Disturbances Flow, SDF, are present. In the NDF the height of the jet remains constant with the distance from the slot exit, while in SDF the jet height increases or decreases without forming vortices.

The structure of the passive scalar field is similar, since it mimics the instantaneous vorticity field, and is similar to the 2D field, [51], as far as the NDF and SDF are concerned, at the two smaller Reynolds numbers, while is somewhat different at the two highest ones. The NDF and SDF, present just downstream the slot exit, are eddy-free regions where the velocity profile remains almost unaltered compared to the slot exit. The length of NDF shrinks with the increasing Reynolds number, as in the experiments, almost disappearing at $\mathrm{Re}=40,000$, in agreement with the classical two-region description. Downstream the NDF and SDF, a region appears with symmetric vortex-pairs at the interface with the stagnant air, where the velocity profile spreads more compared with the NDF and SDF, but the velocity on the centerline remains constant in the Potential Core Region (PCR). The symmetry of the vortex-pairs is typical of the turbulent flow, whereas in the laminar regime the flow field oscillates around the centerline. The vortices growth with the axial distance occupying the entire thickness of the jet, where velocity on the centerline diminishes and PCR ends. At the beginning of the FDR the vortices break, by virtue of the vortex-stretching, in very small structures which form a turbulent wake. This is the main difference with the 2D numerical results, [51], where this region is not present, and the large eddies occupy the entire domain forming an asymmetric pattern.

The turbulent stresses in the NDF and SDF are negligible, in comparison to the viscous ones, at the interface between jet and stagnant air. As far as the Kelvin-Helmholtz Instability (KHI) appears, the mixing begins and the NDF and SDF disappear. The stagnant air is entrained into the vortices and the passive scalar diffuses in these structures. The comparison of the present results with the $2 \mathrm{D}$ ones confirm that, also in presence of a $1 \%$ perturbation of the turbulence intensity, the NDF is present immediately after the slot exit, followed by the SDF.

Figure 1a shows that the NDF is long about $x=h$ at $\mathrm{Re}=5000$, and is followed by the SDF, up to $x=1.5 \mathrm{~h}$, where the height of the jet increases without the formation of vortices. After the SDF, two pairs of vortices appear before the vortex breakdown. Figure $1 \mathrm{~b}$ shows that the length of the NDF at $\mathrm{Re}=10.000$ decreases to $x=0.5 h$, while the total length, NDF and SDF, is $x=0.8 h$. For higher Reynolds numbers, i.e. 20,000 and 40,000, the total lengths of the NDF and SDF are smaller than about $x=0.3 \mathrm{~h}$.

Fig. 1: Instantaneous passive scalar fields. (a) $R e=5000$; (b) $R e=10,000$; (c) $\operatorname{Re}=20,000$; (d) $R e=40,000$. 


\subsection{Mean fields}

The fields of the mean passive scalar, the mean passive scalar variance and the mean turbulent passive scalar flux components, are reported in Figs. 2-6.

Figure 2 shows the mean passive scalar fields for the four Reynolds numbers investigated. Figure 2a shows that the mean passive scalar at $\mathrm{Re}=5,000$ remains unaltered in the URF, compared to the slot exit, up to $x=1.5 \mathrm{~h}$. The length of the URF decreases with the increasing Reynolds number, being approximately long $x=0.8 h$ at $\operatorname{Re}=10,000$, and negligible at $\operatorname{Re}=20,000$ and $\operatorname{Re}=40,000$. Downstream the URF, the mean passive scalar spreading increases considerably, evidencing a triangular region, with the tip on the centerline, where $\Phi=1$, typical of the PCR. The end of the triangular region is clearly visible for all the Reynolds numbers investigated since the vortex-stretching occurs and the degradation of the jet is visible at the center. The passive scalar spreads gradually in the extensive mixing layer, wide about $y=2 h$ at the end of the PCR. A closer look at the mixing layer identifies three different transversal regions: the central one, where $\Phi=1$, a thin low-turbulence sub-layer, where the passive scalar diffuses slowly and the mean passive scalar is around $\Phi=0.85$, and a thick high-turbulence sub-layer, where the passive scalar diffuses strongly. The present 3D mean passive scalar fields show some differences with the 2D counterparts, [51], especially at the two smaller Reynolds numbers investigated.

Fig. 2: Mean passive scalar fields. (a) $\operatorname{Re}=5,000$; (b) $\operatorname{Re}=10,000$; (c) $\operatorname{Re}=20,000$; (d) $\operatorname{Re}=40,000$.

Figure 3 shows the fields of the mean passive scalar variance, $\overline{\phi^{\prime 2}}$. Despite the presence of a $1 \%$ perturbation in the turbulence intensity, the free shear layer shows an initial region where $\overline{\phi^{\prime 2}}$ is null, which corresponds to the URF. A plausible explanation is that on the slot exit, i.e. at the interface between jet and stagnant air, the velocity gradient is so steep that the turbulent stresses are negligible compared to the viscous ones. The numerical results of Fig. 3 show that the length of the region where the mean passive scalar variance is zero decreases with the increase of the Reynolds number, becoming negligible at the two greatest Reynolds numbers. A central triangular region, with null mean passive scalar variance, appears after the URF, which corresponds to the borders of the PCR. Outside the PCR, the passive scalar variance increases in the free shear layer, according to the linear theory on KHI, [73], where a low wave number perturbation is enough to trigger the instabilities at high Reynolds numbers. A comparison of the fields of the mean passive scalar variance, $\overline{\phi^{\prime 2}}$, of Fig. 3, with those of the mean passive scalar of Fig. 2, shows the correspondence between the areas with zero mean passive scalar variance, $\overline{\phi^{\prime 2}}$, and those with $\Phi=1$. The mean passive scalar variance decreases at the end of the PCR assuming smaller values in the FDR, being more uniform at higher Reynolds numbers. The conclusion of the present results is that turbulence affects the mean passive scalar and the momentum in a different way.

Fig. 3: Fields of the mean passive scalar variance. (a) $R e=5,000$; (b) $R e=10,000$; (c) $R e=20,000$; (d) $R e=40,000$. 
Figures 4-6 report the fields of the three components of the mean turbulent passive scalar, which are zero in the URF and in the central region of the PCR, in analogy with the mean passive scalar variance.

Figure 4 presents the axial component of the mean turbulent passive scalar, which is not proportional to the mean axial passive scalar gradient, as the Boussinesq hypothesis would dictate, but with a complex pattern, because regions of positive and negative values are present. After the URF, the mean passive scalar flux is directed downstream in the two buffer regions, respectively at the boundary with the stagnant air and in the PCR, while, at the end of the PCR, is directed upstream. The pattern at the end of the URF is almost symmetrical with respect of the centerline, as result of the instant roller vortices moving downstream, and is always positive because of the high speed. Towards the end of the PCR the roller vortices are closer to the centerline, as shown by Fig. 1, causing a mean passive scalar flux from the far-field upstream the inner roller-vortices. The axial component of the mean turbulent passive scalar flux tends to decrease at the end of the PCR assuming smaller values in the FDR. The present 3D axial component of the mean turbulent passive scalar shows differences with the 2D counterparts, [51]. Firstly, the 3D values are much smaller than the 2D ones, due to the spreading in the z-direction. Secondly, the structure is different around the end of the PCR and after, because of the vortex breakdown, especially at the greater Reynolds numbers.

Fig. 4: Fields of the axial mean turbulent passive scalar flux. (a) $R e=5,000 ;$ (b) $R e=10,000 ;$ (c) $R e=20,000$; (d)

$$
\operatorname{Re}=40,000 \text {. }
$$

Figure 5 presents the transversal component of the mean turbulent passive scalar flux, which is predominant in the mixing region surrounding the PCR and in the FDR, despite the axial flux. The transversal component has greater values than the axial ones because the lateral diffusion is predominant. The fields of the mean turbulent passive scalar flux appear to be jagged, in analogy with the Reynolds stress ones, shown in [39], but, unlike the axial component, they are anti-symmetric with respect to the centerline. The transversal component is directed from the inner part of the jet towards the stagnant air and is always positive in the upper mixing layer, while is always negative in the lower mixing one. The mean transversal component is zero in the URF and in the PCR, being responsible for the spreading of the mean passive scalar. Further on, the mean transversal component is proportional to the mean passive scalar transversal gradient, in agreement with the Boussinesq hypothesis. The transversal component is anti-symmetric, is greater in the mixing region and smaller in the Far-Field Region.

Fig. 5: Fields of the transversal mean turbulent passive scalar flux. (a) $\operatorname{Re}=5,000$; (b) $R e=10,000$; (c) $R e=20,000$;

$$
\text { (d) } R e=40,000 \text {. }
$$

Figure 6 shows the z-component of the mean turbulent passive scalar, which, as the other components and the mean passive scalar variance, is zero in the URF and PCR. The $z$-component has opposite signs above and below the centerline, meaning that the vortices above the centerline roll in the anti-clockwise direction, while those below the centerline roll in the clockwise direction. Furthermore, the z-component 
assumes higher values in the PCR, at the interface with the stagnant fluid, while is more uniform in the FDR, assuming considerably smaller values. This result can be explained by analyzing the vortices in the jet-axes normal plane. Just downstream the slot exit the gradients in the transversal direction are as high as well as the mass transfer. At the end of the PCR, the vortex breakdown occurs and the jet becomes axisymmetric assuming more uniform and smaller values in the FDR. From the fields of the mean turbulent passive scalar of Figs. 4-6 it is possible to distinguish between two different behaviors in the NFR, plus a third one downstream the far-field, which cannot be captured by the 2D simulations, [51], because of the lack of vortex stretching.

Fig. 6: Fields of the z-direction mean turbulent passive scalar flux. (a) $\operatorname{Re}=5,000$; (b) $R e=10,000$; (c) $R e=20,000$; (d) $R e=40,000$.

The turbulent Prandtl or Schmidt number, $P r_{T}=S c_{T}$, is evaluated with Eq. 12 and is reported in Fig. 7, where is indicated as $S c_{T}$. The $P r_{T}=S c_{T}$, is zero in most of the central part of the URF and PCR, where mass transfer does not occur, it is increasing at the PCR interface, where the jet mixes with the stagnant fluid and the heat/mass transfer increases. In the mixing region the passive scalar diffuses less than the momentum and the $P r_{T}=S c_{T}$ becomes greater than 1. At the end of the PCR, the $P r_{T}=S c_{T}$ decreases, assuming smaller but uniform values in the FDR, except at the interface with the stagnant fluid, because of the higher velocity gradient.

Fig. 7: Turbulent Prandtl or Schmidt number. (a) $R e=5,000$; (b) $R e=10,000$; (c) $R e=20,000$; (d) $R e=40,000$.

\section{Theoretical results for the mean solutions in the FDR.}

Despite the presence of a $1 \%$ perturbation in the turbulence intensity on the slot exit, the flow continues to be mostly 2D in the URF and PCR, and the theoretical results for the passive scalar spreading, derived in [51], are confirmed by the present 3D simulations, also if they are not shown here for sake of brevity.

A new theory for the FDR is proposed in this section with comparisons with the 3D numerical results. The Fully Developed Region (FDR) starts downstream the point where turbulence has penetrated into the axis. Downstream the PCR the mean passive scalar on the centerline starts decreasing with the axial coordinate up to a point where is negligible. The iso-passive scalar curves in the FDR, derived from the 3D numerical simulations, are reported in Fig. 8. The slope of the iso-passive scalar curves in the FDR is always positive because turbulence has fully penetrated the jet, which is the characteristic of the FDR.

Fig. 8: Mean passive scalar iso-curves in FDR. $\Phi=0.1$, data (•), regression (-). $\Phi=0.4$, data (०), regression (--). $\Phi=0.7$, data (x), regression (....). $\Phi=0.9$, data ( () , regression (--). (a) $\operatorname{Re}=5000$; (b) $\operatorname{Re}=10,000$; (c) $\operatorname{Re}=$ 20,000; (d) $\operatorname{Re}=40,000$.

Tollmien [23] proposed the following self-similar variable, $\zeta$, and turbulent viscosity, $v_{T}, \backslash$ 
$\zeta=\frac{1}{\sqrt[3]{2 c^{2}}} \frac{y}{\left(x-L_{s}\right)}$

$v_{T}=c^{2} \cdot\left(x-L_{s}\right)^{2} \cdot\left|\frac{\partial U}{\partial y}\right|$

leading to the following ordinary differential equation

$\frac{d}{d \zeta}\left(\left|\frac{d^{2} f}{d \zeta^{2}}\right| \frac{d^{2} f}{d \zeta^{2}}+f \frac{d f}{d \zeta}\right)=0$

Görtler [24] proposed the following self-similar variable, $\xi$, and turbulent viscosity, $v_{T}$,

$\xi=\frac{\sigma}{4 g} \frac{y}{\left(x-L_{s}\right)}$

$v_{T}=g \cdot U_{c} \cdot\left(x-L_{s}\right)$

leading to

$$
f(\xi)=\tanh (\xi)
$$

In both the theories $[23,24]$ the axial velocity at the centerline is $U_{c}=\frac{n}{\sqrt{x-L_{s}}}$, while the constant $\sigma$ is function of the Reynolds number. Abramovich [22] choose values of $\sigma$ spanning between 0.09 and 0.12 and giving, as result, $U / U_{c}(\eta=1)=0.5$, while $\sigma$ spans between 0.06 and 0.1 in [39].

From the mean passive scalar budget it is obtained that, in analogy with the momentum, the time derivative, the molecular and axial turbulent diffusion are negligible. Therefore, the equation of the passive scalar transport reduces to

$U \frac{\partial \Phi}{\partial x}+V \frac{\partial \Phi}{\partial y}=\frac{\partial}{\partial y}\left(\frac{v_{T}}{S c_{T}} \frac{\partial \Phi}{\partial y}\right)$

In analogy with the momentum, the mean passive scalar, spreading in the FDR, is not self-similar but depends on the axial coordinate, and is supposed to be the product of its value on the centerline, $\Phi_{c}$, which decays with the distance, and a "scaled" passive scalar, $\hat{\phi}$, which is self-similar. Replacing this assumption into Eq. (19) it is obtained 
$\hat{\phi} U \frac{1}{\Phi_{c}} \frac{d \Phi_{c}}{d x}+U \frac{\partial \hat{\phi}}{\partial x}+V \frac{\partial \hat{\phi}}{\partial y}=\frac{\partial}{\partial y}\left(\frac{v_{T}}{S c_{T}} \frac{\partial \hat{\phi}}{\partial y}\right)$

By using the results of [23] for the momentum, Eq. (20) becomes

$$
\frac{d}{d \zeta}\left(\left|\frac{d^{2} f}{d \zeta^{2}}\right| \frac{1}{S c_{T}} \frac{d \hat{\phi}}{d \zeta}\right)+f(\zeta) \frac{d \hat{\phi}}{d \zeta}-2 \hat{\phi} \frac{d f}{d \zeta}\left(x-L_{s}\right) \frac{1}{\Phi_{c}} \frac{d \Phi_{c}}{d x}=0
$$

while, using those of [24], Eq. (20) becomes

$$
\frac{d}{d \xi}\left(\frac{1}{S c_{T}} \frac{d \hat{\phi}}{d \xi}\right)+2 f(\xi) \frac{d \hat{\phi}}{d \xi}-4 \hat{\phi} \frac{d f}{d \xi}\left(x-L_{s}\right) \frac{1}{\Phi_{c}} \frac{d \Phi_{c}}{d x}=0
$$

The analysis of each member of Eqs. (21-22) shows that, in order to satisfy the self-similarity of $\hat{\phi}$ the following two conditions are required: (i) the turbulent Prandtl or Schmidt number should be a selfsimilar function (in analogy with the PCR); (ii) the average passive scalar on the axis should satisfy the following equation

$$
\left(x-L_{s}\right) \frac{1}{\Phi_{c}} \frac{d \Phi_{c}}{d x}=K
$$

As far as the $P r_{T}, S c_{T}$ is concerned, the analysis shows that it is approximately constant and equal to 0.7 in the FDR, which satisfies the self-similarity property. This conclusion is in agreement with the theory of the FDR for circular jets, where the passive scalar is proportional to the axial velocity through a powerlaw relationship, with exponent $P r_{T}, S c_{T}[53,54]$.

As far as the mean passive scalar on the centerline is concerned, Eq. (23) leads to

$$
\Phi_{c}(x)=\Phi_{c, 1}\left|x-L_{s}\right|^{K}
$$

By analyzing the numerical results of the passive scalar decay along the centerline, it is found that $\mathrm{K}=-0.5$. Therefore, the passive scalar spreading, using the results of [23] for the momentum in Eq. (21), becomes

$$
\frac{d}{d \zeta}\left(\left|\frac{d^{2} f}{d \zeta^{2}}\right| \frac{1}{S c_{T}} \frac{d \hat{\phi}}{d \zeta}+f \hat{\phi}\right)=0
$$

The integration of Eq. (25), with $\lim _{\zeta \rightarrow \infty} \frac{d \hat{\phi}}{d \zeta}=0 \Rightarrow C_{T}=0$, gives the stream function expression

$$
\hat{\phi}(\zeta)=\exp \left(-\int_{0}^{\zeta} S c_{T} f\left|\frac{d^{2} f}{d \zeta^{2}}\right|^{-1} d t\right)
$$

On the other hand, by using the results of [24] for the momentum, Eq. (27) becomes 
$\frac{d}{d \xi}\left(\frac{1}{S c_{T}} \frac{d \hat{\phi}}{d \xi}+2 f \hat{\phi}\right)=0$

The integration of Eq. (27), with $\lim _{\xi \rightarrow \infty} \frac{d \hat{\phi}}{d \xi}=0 \Rightarrow C_{G}=0$, gives the following expression of the average passive scalar expression

$\hat{\phi}(\xi)=\frac{1}{(\cosh (\xi))^{2 S c_{T}}}$

The modulus of the transversal mass flux per unit area, using the results of [23] and [24], can be finally obtained

$$
\begin{aligned}
& \left|J_{y}\right|=\frac{v_{T}}{S c_{T}}\left|\frac{\partial \Phi}{\partial y}\right|=\frac{\sigma}{2} \frac{n \Phi_{c, 1}}{\left(x-L_{s}\right)} f(\zeta) \exp \left(-\int_{0}^{\zeta} S c_{T} f(\zeta)\left|\frac{d^{2} f}{d \zeta^{2}}\right|^{-1} d t\right) \\
& \left|J_{y}\right|=\frac{v_{T}}{S c_{T}}\left|\frac{\partial \Phi}{\partial y}\right|=\frac{\sigma}{2} \frac{n \Phi_{c, 1}}{\left(x-L_{s}\right)} \frac{\sinh (\xi)}{(\cosh (\xi))^{2 S c_{T}+1}}
\end{aligned}
$$

According to both theories, mass transfer decreases with the axial distance from the slot, it is zero in the stagnant air and on the axis, and has a maximum in the mixing region. The comparison between the mean passive scalar profiles, obtained with the present 3D numerical approach and scaled by its axial value, and the theoretical results at different Reynolds numbers is shown in Figure 9. The passive scalar, $\Phi / \Phi_{c}$, is plotted versus $y / b$, where $b=\sigma_{A}\left(x-L_{s}\right)$, and $\sigma_{A}$ is the value given in [22] for the constant $\sigma$, such that $y / b(x)=1 \Rightarrow U / U_{c}=0.5$. The theoretical profiles are compared with the 3D LES numerical results at several axial distances, in order to show that the hypothesis of self-similarity is verified.

The agreement between the 3D numerical results and the two theories is quite good, even at high Reynolds numbers. In general, the theoretical solution, based on the momentum spreading of [23], and called Tollmien-like for the FDR (TFDR), fits better the results than that based on the momentum spreading of [24], and called Görtler-like for the FDR (GFDR). Both theories describe well enough the average passive scalar spreading in the range of $\Phi / \Phi_{c} \in[0.3,1]$. The GFDR solution overestimates the passive scalar for $\Phi / \Phi_{c}<0.3$, while the TFDR solution is in good agreement or slightly underestimates it. An interesting facet of the mean passive scalar profiles is that $y / b(x)=1 \Rightarrow \Phi / \Phi_{c} \approx 0.6$. The scaled mean passive scalar profile is in first approximation linear function of $y / b(x)$, it becomes more concave towards the jet axis and convex in the stagnant air. 
Fig. 9: Mean passive-scalar profile in the FDR. Numerical and “Tollmien-like for the FDR" (TFDR) and "Görtlerlike for the FDR" (GFDR) theoretical results. (a) $R e=5,000 ;$ (b) $R e=10,000$; (c) $R e=20,000 ;$ (d) $R e=40,000$.

\section{Conclusions}

The present paper investigates the passive scalar spreading in the near-field and far-field regions of the evolution of turbulent rectangular submerged free jets of air with $\operatorname{Pr}=S_{C}=1$, by means of a 3D LES numerical simulation approach and a theoretical analysis. A series of Large Eddy Simulations (LES) are carried out at four Reynolds numbers, namely 5000, 10,000, 20,000 and 40,000. A perturbation of $1 \%$ in the turbulence intensity of the jet is added on the slot exit.

The 3D LES numerical simulations present instantaneous and mean passive scalar profiles, which are more general than the 2D counterparts, as well as the 3D fields of the passive scalar variance and of the three components of the turbulent passive scalar fluxes. The present 3D theoretical analysis follows the previous 2D one, [51], which investigated only the near-field region (NFR) of jets, finding that the passive scalar spreading in the mean Undisturbed Region of Flow (URF) and in the Potential Core Region (PCR) follows a self-similar behavior. Further on, the flow continues to be mostly 2D in the instantaneous Negligible Disturbances Flow (NDF) and Small Disturbances Flow (SDF).

The turbulent Prandtl or Schmidt number is evaluated numerically in the mean undisturbed region of flow, URF, in the potential core region, PCR, and in the fully developed region, FDR, showing new interesting features. The turbulent Prandtl or Schmidt number is zero in most part of the URF, and in the triangular region of the PCR, while in the FDR is constant and approximately equal to 0.7, meaning that turbulence spreads the passive scalar more than the momentum. These conclusions are another strong confirmation of the new structure in the flow evolution of turbulent rectangular submerged free jets of air, with the instantaneous NDF and SDF, or the mean URF, which are present before the PCR and FDR.

The present work uses two new self-similar mathematical models to describe the mean passive scalar spreading in the FDR, by using the expressions of the momentum spreading proposed in [23, 24]. The derivation of the two theoretical models for the FDR is an important result as this region has virtually no end, and therefore the model developed has a large domain of applicability. Furthermore, the model proposed represents an advancement of the Gaussian Plume Model (GPM) of circular jets, which is derived under the hypothesis of constant eddy momentum and passive scalar diffusivities. The methodology used in this work can be applied to study other relevant applications involving heat and mass transfer.

\section{References}

[1] O. Reynolds, On the extent and action of the heating surface for steam boilers, Proc. Lit. Phil. Soc., Manchester, 14 (1874) 7-12. 
[2] J. Kestin, L. N. Persen, Application of Schmidt's Method to the Calculation of the Spalding's function and of the Skin-friction Coefficient in Turbulent Flow, International Journal of Heat and Mass Transfer, 5 (34) (1962) 143-152.

[3] H.W. Kropholler, A.D. Carr, The prediction of heat and mass transfer coefficients for turbulent flows in pipes at values of the Prandtl and Schmidt number, International Journal of Heat and Mass Transfer, 5 (1962) 1191-1205.

[4] J. Kestin, P.D. Richardson, Heat Transfer across Turbulent, incompressible boundary layers, International Journal of Heat and Mass Transfer, 6 (1963) 147-189.

[5] J. Blom, D. A. De Vries, On the value of turbulent Prandtl number, in Heat and Mass Transfer, Proc. 3d All Soviet Union Conf., Edited by A. V. Lykov and B. M. Smol'skii, 1 (1968) 147-154, Energiya, Moscow.

[6] D.B. Spalding, Concentration fluctuations in a round turbulent free jet. Chemical Engineering Science, 26 (1) (1971) 95-107.

[7] B.A. Kader, A.M. Yaglom, Heat and Mass Transfer Laws for Fully Turbulent Wall Flows, International Journal of Heat and Mass Transfer, 15 (1972) 2329-2351.

[8] A.J. Reynolds, The Prediction of Turbulent Prandtl and Schmidt numbers, International Journal of Heat and Mass Transfer, 18 (1975) 1055-1069.

[9] A.J. Reynolds, The Variation of Turbulent Prandtl and Schmidt Numbers in Wakes and Jets, International Journal of Heat and Mass Transfer, 19 (1976) 757-764.

[10] M. Jischa, H.B. Rieke, About the Prediction of Turbulent Prandtl and Schmidt Numbers from Modelled Transport Equations, International Journal of Heat and Mass Transfer, 22 (1979) 1547-1555.

[11] F. Gori, M.A. El Hadidy, D.B. Spalding, Numerical Prediction of the Heat Transfer to Low-Prandtl Number Fluids, Numerical Heat Transfer, 2, 441-454, 1979.

[12] S. Faggiani, F. Gori. Influence of Stream-wise Molecular Heat Conduction on the Heat Transfer Coefficient for Liquid Metals in Turbulent Flow between Parallel Plates, Journal of Heat Transfer, ASME, 102 (2) (1980) 292-296.

[13] M.A. El Hadidy, F. Gori, D.B. Spalding, Further Results on the Heat Transfer to Low-Prandtl Number Fluids in Pipes, Numerical Heat Transfer, 5 (1982) 107-117.

[14] P. Givi, J.L. Ramos, On the calculation of heat, mass and momentum transport in coaxial jets and mixing layers, International Communications in Heat Mass Transfer, 12 (1985) 323-336.

[15] L.P. Chua, R.A. Antonia, Turbulent Prandtl number in a circular jet, International Journal of Heat and Mass Transfer, 33-2 (1990) 331-339.

[16] Q. Chen, V. Modi, Mass Transfer in Turbulent Impinging Slot Jets, International Journal of Heat and Mass Transfer, 42 (1999) 873-887.

[17] G. He, G. Yanhu, A. T. Hsu, The effect of Schmidt number on turbulent scalar mixing in a jet-incrossflow, International Journal of Heat and Mass Transfer 42 (20) (1999) 3727-3738. 
[18] Y. Tominaga, S. Ted, Turbulent Schmidt numbers for CFD analysis with various types of flow field, Atmospheric Environment, 41 (37) (2007) 8091-8099.

[19] F. Schwertfirm, M. Manhart, DNS of passive scalar transport in turbulent channel flow at high Schmidt numbers, International Journal of Heat and Mass Transfer, 28 (2007) 1204-1214.

[20] M. van Reeuwijk, M. Hadziabdic, Modelling high Schmidt number turbulent mass transfer, International Journal of Heat and Fluid Flow, 51 (2015) 42-49.

[21] M. L. Albertson, Y. B. Dai, R. A. Jensen, H. Rouse, Diffusion of Submerged Jets, Trans. Am. Soc. Civ. Eng., 410 (115) 1 (1950) 639-64.

[22] G.N. Abramovich, On the Deformation of the Rectangular Turbulent Jet Cross-Section, International Journal of Heat and Mass Transfer, 25 (12) (1982) 1885-1894.

[23] W. Tollmien, Calculation of Turbulent Expansion Processes, Zeitschrift Für Angewandte Mathematik Und Mechanik, 6 (6) (1926) 468-78.

[24] H. Görtler, Berechnung von Aufgaben Der Freien Turbulenz Auf Grund Eines Neuen Naherungsansatzes, Zeitschrift Für Angewandte Mathematik Und Mechanik, 22 (5) (1942) 244-254.

[25] E. Forthmann, Turbulent Jet Expansion, NACA-TM-789, 1936.

[26] B.G. Van der Hegge Zijnen, Measurements of the Distribution of Heat and Matter in a Plane Turbulent Jet of Air, Appl. Sci. Res., 7 (4) (1958) 277-292.

[27] H.W. Liepmann, J. Laufer, Investigations of Free Turbulent Mixing, NACA Technical Note, 1947.

[28] A.A. Sfeir, The Velocity and Temperature Fields of Rectangular Jets, International Journal of Heat and Mass Transfer, 19 (11) (1976) 1289-97.

[29] F. Gori, F. De Nigris, E. Nino, Fluid Dynamics Measurements and Optical Visualization of the Evolution of a Submerged Slot Jet of Air, Proceedings of the 12th International Heat Transfer, Editors: J. Taine, Editions Elsevier, Grenoble, France, 2 (2002) 303-308.

[30] F. Gori, I. Petracci, Fluid Dynamics Measurements in a Submerged Free Slot Jet of Air, Proceedings of the 14th International Symposium on Transport Phenomena, Bali, Indonesia, 6-10th July, (2003) 491-496.

[31] F. Gori, E. Nino, Fluid Dynamics Measurements and Flow Visualizations of a Free Slot Jet of Air, American Society of Mechanical Engineers, Fluids Engineering Division (Publication) FED, 259 (2003) 187-192.

[32] F. Gori, I. Petracci, Fluid Dynamics Measurements and Numerical Simulations around a Circular Cylinder Impinged by a Submerged Slot Jet of Air, American Society of Mechanical Engineers, Fluids Engineering Division (Publication) FED, 259 (2003) 179-185.

[33] F. Gori, E. Nino, I. Petracci, Shadowgraph Visualizations of a Submerged Free Slot Jet of Air, International Journal of Heat and Technology, 25 (1) (2007) 157-164.

[34] F. Gori, I. Petracci, M. Angelino, Flow Evolution of a Turbulent Submerged Two-Dimensional Rectangular Free Jet of Air. Average Particle Image Velocimetry (PIV) Visualizations and Measurements, International Journal of Heat and Fluid Flow, 44 (2013) 764-775. 
[35] F. Gori, I. Petracci, M. Angelino, Influence of the Reynolds number on the instant flow evolution of a turbulent rectangular free jet of air, International Journal of Heat and Fluid Flow, 50 (2014) 386-401.

[36] F. Gori, I. Petracci, Heat Transfer Measurements and Numerical Simulations in the Cooling of a Circular Cylinder by a Slot Jet of Air, American Society of Mechanical Engineers, Heat Transfer Division, (Publication) HTD, 374 (1) (2003) 3-9.

[37] F. Gori, M. Angelino, A. Boghi, I. Petracci, Preliminary Numerical Solutions of the Evolution of Free Jets, ASME International Mechanical Engineering Congress and Exposition, Proceedings (IMECE), 7 (2012) 463-469.

[38] A. Boghi, M. Angelino, F. Gori, Numerical evidence of an undisturbed region of flow in a turbulent rectangular submerged free jet, Numerical Heat Transfer, Part A: Applications, 70 (1) (2016): 14-29.

[39] M. Angelino, A. Boghi, F. Gori, Numerical solution of three-dimensional rectangular submerged jets with evidence of the undisturbed region of flow, Numerical Heat Transfer, Part A: Applications, 70 (8) (2016) 815-830.

[40] I.V. Derevich, Influence of internal turbulent structure on intensity of velocity and temperature fluctuations of particles, International Journal of Heat and Mass Transfer, 44 (23) (2001) 4505-4521.

[41] J.S. Wu, Y.J. Liu, H.J. Sheen, Effects of ambient turbulence and fuel properties on the evaporation rate of single droplets, International Journal of Heat and Mass Transfer, 44 (24) (2001) 4593-4603.

[42] J.J. Nijdam, T.A.G. Langrish, D.F. Fletcher, Assessment of an Eulerian CFD model for prediction of dilute droplet dispersion in a turbulent jet, Applied Mathematical Modelling 32 (12) (2008) 2686-2705.

[43] F. Gori, I. Petracci, Influence of turbulence on heat transfer upon a cylinder impinged by a slot jet of air, Applied Thermal Engineering, 49 (2012) 106-117.

[44] F. Gori, I. Petracci, Influence of screen solidity ratio on heat transfer upon a cylinder impinged by a rectangular jet, International Journal of Heat and Mass Transfer, 81 (2015) 19-27.

[45] K. Chen, L. Miao, A Lorentz Force Powered Controllable Micro-Jet Injection System, Computer Science and Engineering Technology (CSET2015), Medical Science and Biological Engineering (MSBE2015), Proceedings of the 2015 International Conference on CSET and MSBE, World Scientific, (2015) 170.

[46] J.J. Yoh, H. Jang, M. Park, T. Han, J. Hah, A bio-ballistic micro-jet for drug injection into animal skin using a Nd: YAG laser, Shock Waves, 26 (1) (2016) 39-43.

[47] D. Hadryś, T. Węgrzyn, J. Piwnik, Ł. Wszołek, D. Węgrzyn, Compressive Strength of Steel Frames after Welding with Micro-Jet Cooling, Archives of Metallurgy and Materials, 61 (1) (2016) 123-126.

[48] T. Wegrzyn, J. Piwnik, B. Lazarz, W. Tarasiuk, Mechanical properties of shaft surfacing with micro-jet cooling, Mechanics, 21 (5) (2015) 419-423.

[49] M.A. Islam, Einstein-Smoluchowski diffusion equation: a discussion, Physica Scripta, 70 (2-3) (2004) 120. 
[50] E. Cunningham, On the velocity of steady fall of spherical particles through fluid medium, Proceedings of the Royal Society of London, Series A, Containing Papers of a Mathematical and Physical Character, 83 (563) (1910) 357-365.

[51] A. Boghi, I. Di Venuta, F. Gori, Passive scalar diffusion in the near field region of turbulent rectangular submerged free jets, International Journal of Heat and Mass Transfer, 112 (2017) 1017-1031.

[52] I. Di Venuta, I. Petracci, M. Angelino, A. Boghi, F. Gori, Numerical simulation of mass transfer and fluid flow evolution of a rectangular free jet of air, International Journal of Heat and Mass Transfer, 117 (2018) 235-251.

[53] J.O. Hinze, Turbulence, McGraw-Hill, New York (1975).

[54] A.A. Townsend, The structure of turbulent shear flow, Cambridge University Press, 1980.

[55] R. Kumar, A. Dewan, URANS computations with buoyancy corrected turbulence models for turbulent thermal plume, International Journal of Heat and Mass Transfer, 72 (2014) 680 - 689.

[56] F. Gori, L. Bossi, On the Cooling Effect of an Air Jet along the Surface of a Cylinder, International Communications in Heat and Mass Transfer, 27 (5) (2000) 667-676.

[57] F. Gori, L. Bossi, Optimal Slot Height in the Jet Cooling of a Circular Cylinder, Applied Thermal Engineering, 23 (7) (2003) 859-870.

[58] F. Gori, I. Petracci, Local Heat Transfer on a Circular Cylinder Impinged by a Submerged Slot Jet of Air,

Eurotherm Seminar 74, March 23-26, Eindhoven, The Nederlands, (2003) 63-69.

[59] F. Gori, G. Pacchiarotti, I. Petracci, Pulsating Jets Cooling Circular Cylinders, American Society of Mechanical Engineers, Process Industries Division (Publication) PID, 8 (2003) 153-159.

[60] F. Gori, I. Petracci, M. Angelino, Experimental and Numerical Heat Transfer on a Cylinder Cooled by Two Rectangular Jets of Different Heights, ASME International Mechanical Engineering Conference and Exposition, Proceedings (IMECE), 7 (D) (2012) 2679-2688.

[61] F. Gori, I. Petracci, Enhancement of Heat Transfer on a Cylinder by the Turbulence of a Slot Jet: Preliminary Results, Proceedings of ASME-ATI-UIT 2010 Conference on Thermal and Environmental Issues in Energy Systems, 16-19 May, Sorrento, Italy, II, (2010) 1383-1388.

[62] F. Gori, I. Petracci, On the Effect of the Slot Height in the Cooling of a Circular Cylinder with a Rectangular Jet, International Communications in Heat and Mass Transfer, 48 (2013) 8-14.

[63] F. Gori, F. De Nigris, E. Pippione, G. Scavarda, Cooling of Finned Cylinders by a Jet Flow of Air, American Society of Mechanical Engineers, Process Industries Division (Publication), PID, 7 (2002) 117122.

[64] F. Gori, M. Borgia, A. Doro Altan, Cooling of a Finned Cylinder by a Jet Flow of Air, American Society of Mechanical Engineers, Process Industries Division (Publication) PID, 8 (2003) 147-152.

[65] F. Gori, M. Borgia, A. Doro Altan, M. Mascia, I. Petracci, Cooling of a Finned Cylinder by a Jet Flow of Air, ASME Journal of Heat Transfer, 127 (12) (2005) 1416-1421. 
[66] F. Gori, M. Mascia, I. Petracci, Air cooling of a finned cylinder with slot jets of different height, International Journal of Thermal Sciences, 50 (2011) 1583-1593.

[67] I. Petracci, L. Manni, F. Gori, Numerical simulation of the optimal spacing for a radial finned tube cooled by a rectangular jet. I-Average thermal results, International Journal of Thermal Sciences, 104 (2016) 54-67.

[68] F. Gori, L. Bossi, Cooling of Two Cylinders in a Row by a Slot Jet of Air, International Journal of Transport Phenomena, 4 (2002) 245-256.

[69] F. Gori, I Petracci, V. Tedesco, Cooling of two smooth cylinders in row by a slot jet of air with low turbulence, Applied Thermal Engineering, 27 (14-15) (2007) 2415-2425.

[70] F. Gori, P. Coppa, Circumferential variation of heat transfer on three circular cylinders cooled by a slot jet of air, International Journal of Heat and Technology, 16 (2) (1998) 63-69.

[71] F. Gori, L. Bossi, On the optimal configuration of three cylinders in a row cooled by a slot jet of air, American Society of Mechanical Engineers. Heat Transfer Division (Publication), Fundamentals of singlephase convective heat transfer, HTD, 366 (2000) 39-44.

[72] P. Moin, K. Squires, W. Cabot, and S. Lee, A dynamic sub-grid-scale model for compressible turbulence and scalar transport, Phys. Fluids 3 (1991) 2746.

[73] S. Chandrasekhar, Hydrodynamic and Hydromagnetic Stability, Clarendon Press, Oxford University Press, 1961. 


\section{CAPTIONS TO FIGURES}

Fig. 1: Instantaneous passive scalar fields. (a) $\operatorname{Re}=5000$; (b) $\operatorname{Re}=10,000$; (c) $\operatorname{Re}=20,000$; (d) $\operatorname{Re}=40,000$.

Fig. 2: Mean passive scalar fields. (a) $\operatorname{Re}=5,000$; (b) $\operatorname{Re}=10,000$; (c) $\operatorname{Re}=20,000$; (d) $\operatorname{Re}=40,000$.

Fig. 3: Fields of the mean passive scalar variance. (a) $\operatorname{Re}=5,000$; (b) $\operatorname{Re}=10,000$; (c) $\operatorname{Re}=20,000$; (d) $\operatorname{Re}=$ 40,000.

Fig. 4: Fields of the axial mean turbulent passive scalar flux. (a) $\operatorname{Re}=5,000$; (b) $\operatorname{Re}=10,000$; (c) $\operatorname{Re}=$ 20,000; (d) $\operatorname{Re}=40,000$.

Fig. 5: Fields of the transversal mean turbulent passive scalar flux. (a) $\operatorname{Re}=5,000$; (b) $\operatorname{Re}=10,000$; (c) $\operatorname{Re}=$ 20,000; (d) $\operatorname{Re}=40,000$.

Fig. 6: Fields of the z-direction mean turbulent passive scalar flux. (a) $\operatorname{Re}=5,000$; (b) $\operatorname{Re}=10,000$; (c) $\operatorname{Re}=$ 20,000; (d) $\operatorname{Re}=40,000$.

Fig. 7: Turbulent Prandtl or Schmidt number. (a) $\operatorname{Re}=5,000$; (b) $\operatorname{Re}=10,000$; (c) $\operatorname{Re}=20,000$; (d) $\operatorname{Re}=$ 40,000.

Fig. 8: Mean passive scalar iso-curves in FDR., data (•), regression (-). , data (०), regression (-.-). , data (x), regression (....). data $(\diamond)$, regression (--). (a) $\operatorname{Re}=5000$; (b) $\operatorname{Re}=10,000$; (c) $\operatorname{Re}=20,000$; (d) $\operatorname{Re}=40,000$.

Fig. 9: Mean passive-scalar profile in the FDR. Numerical and "Tollmien-like for the FDR" (TFDR) and “Görtler -like for the FDR” (GFDR) theoretical results. (a) Re = 5,000; (b) Re = 10,000; (c) Re = 20,000; (d) $\operatorname{Re}=40,000$. 
(a)

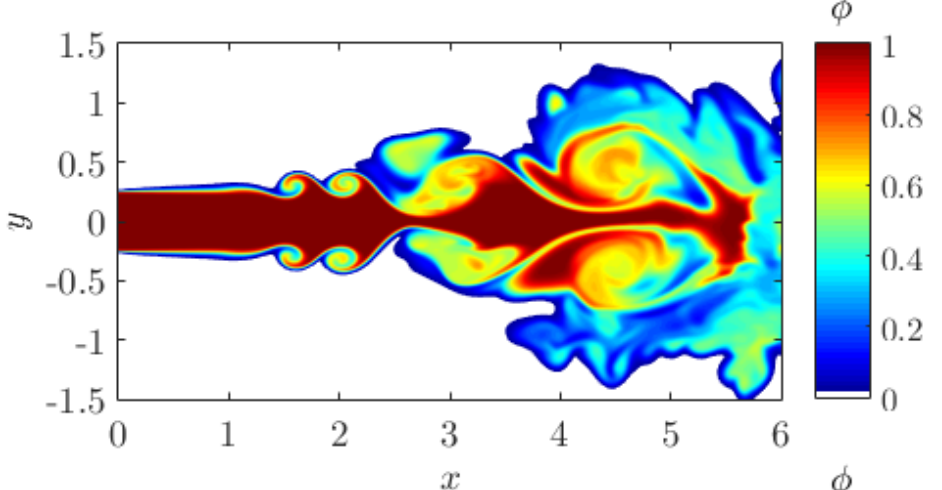

(b)

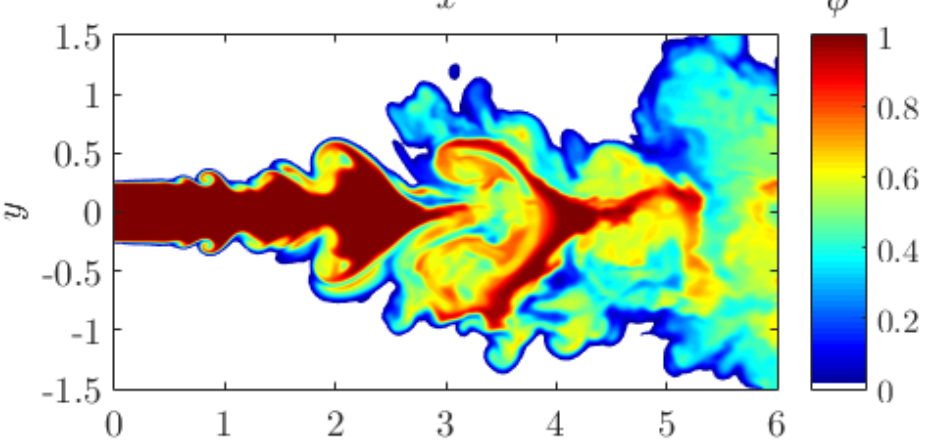

(c)

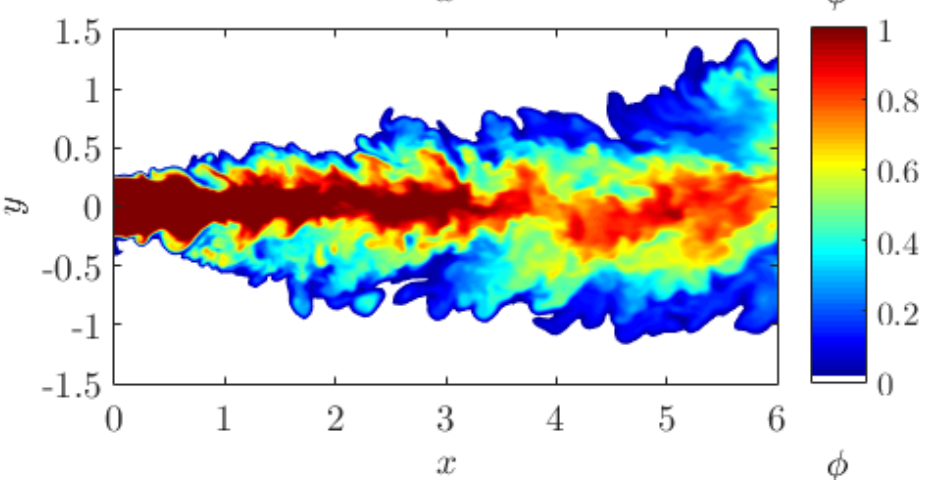

(d)

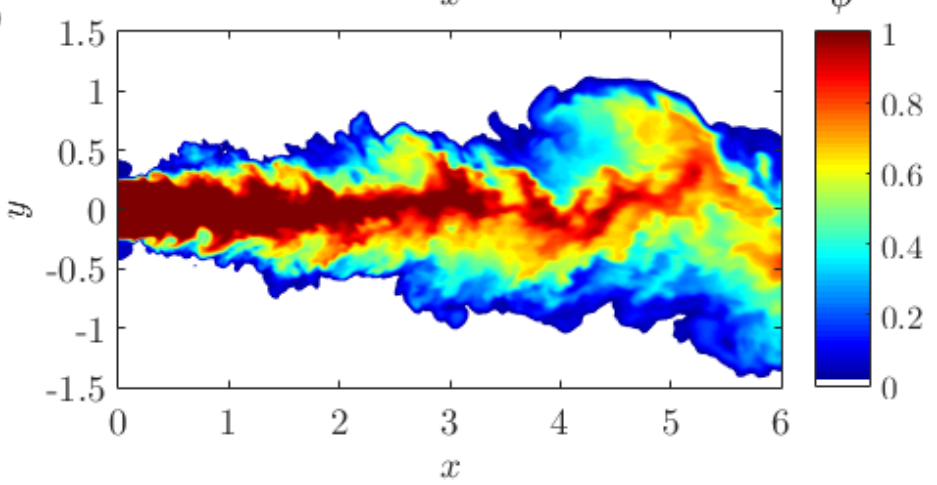

Fig. 1: Instantaneous passive scalar fields. (a) $R e=5000$; (b) $R e=10,000$; (c) $\operatorname{Re}=20,000$; (d) $R e=40,000$. 
(a)

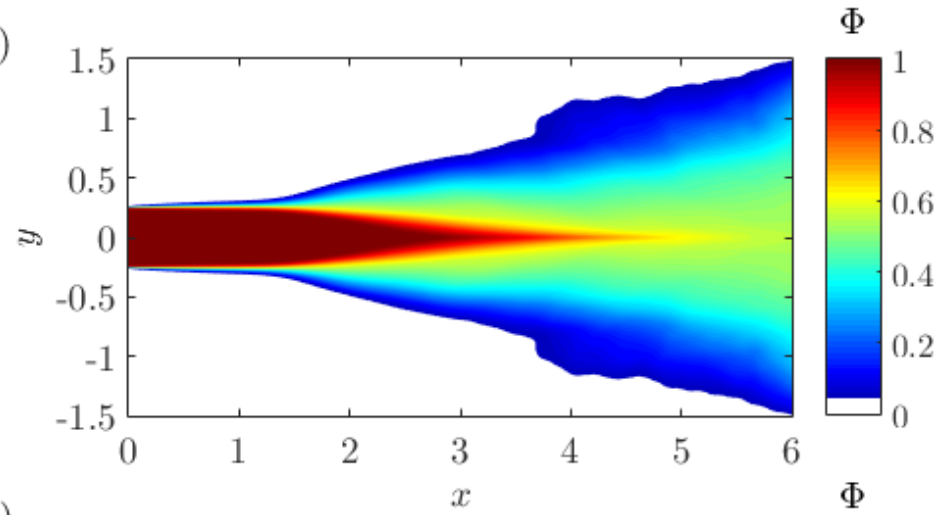

(b)

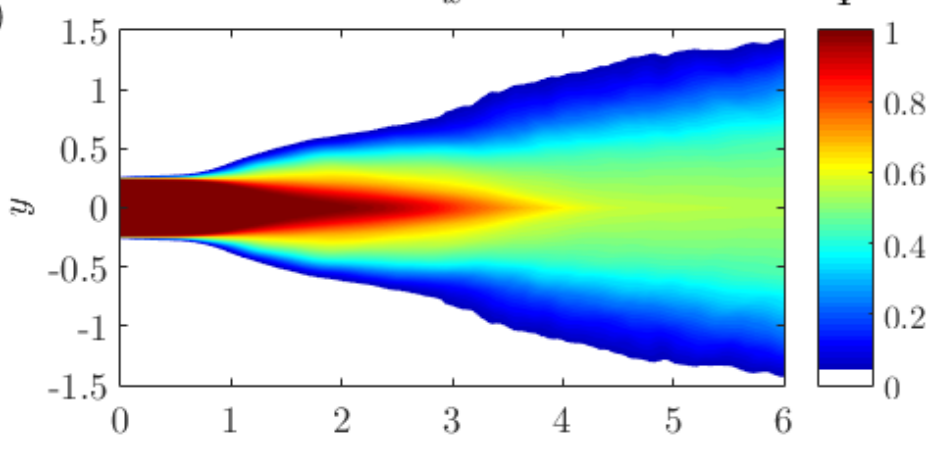

(c)

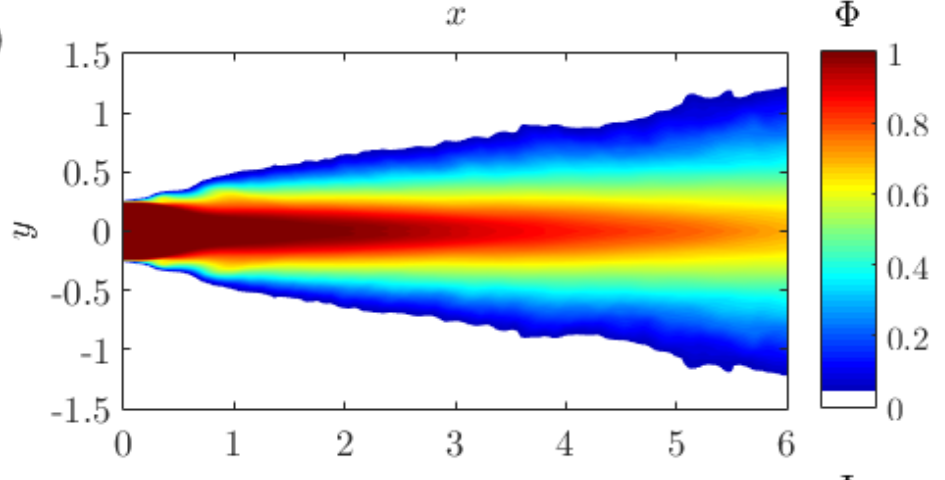

(d)

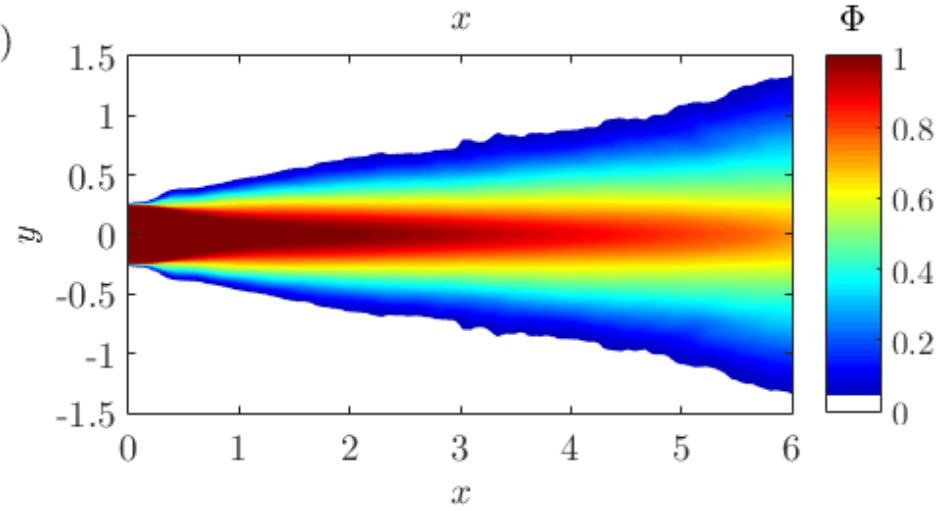

Fig. 2: Mean passive scalar fields. (a) $\operatorname{Re}=5,000$; (b) $\operatorname{Re}=10,000$; (c) $\operatorname{Re}=20,000$; (d) $\operatorname{Re}=40,000$. 
(a)

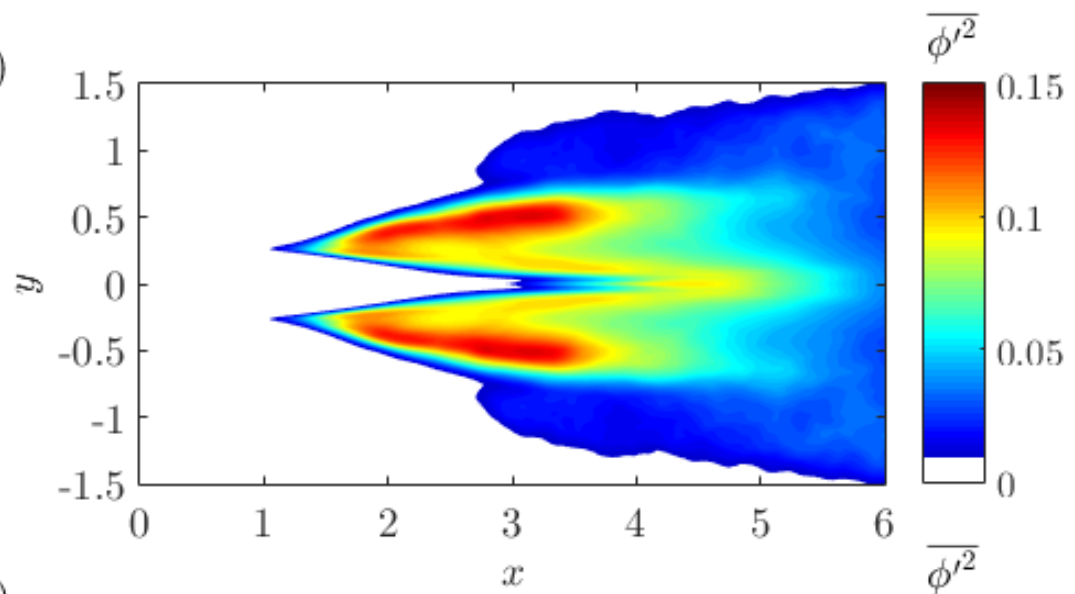

(b)

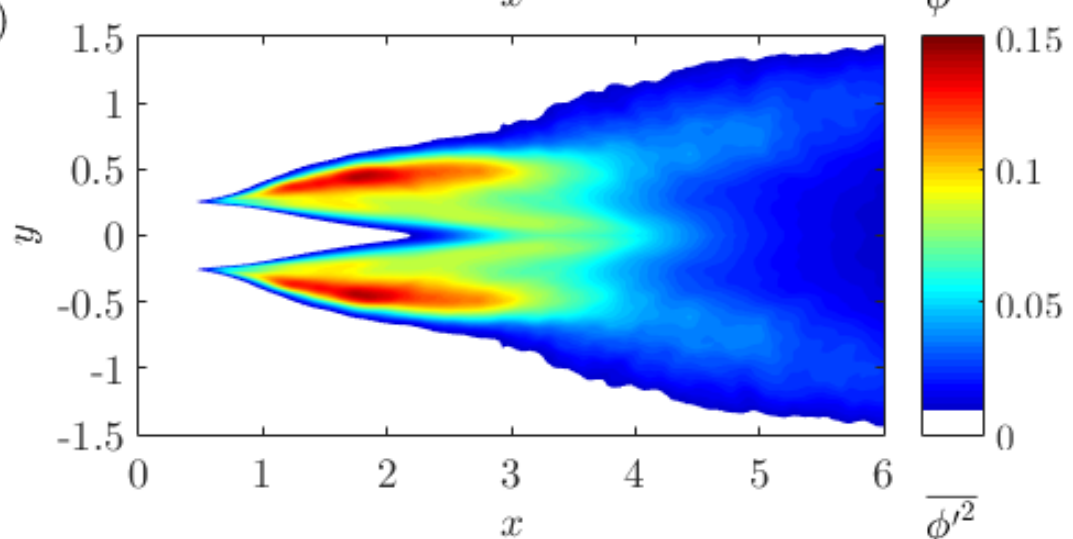

(c)

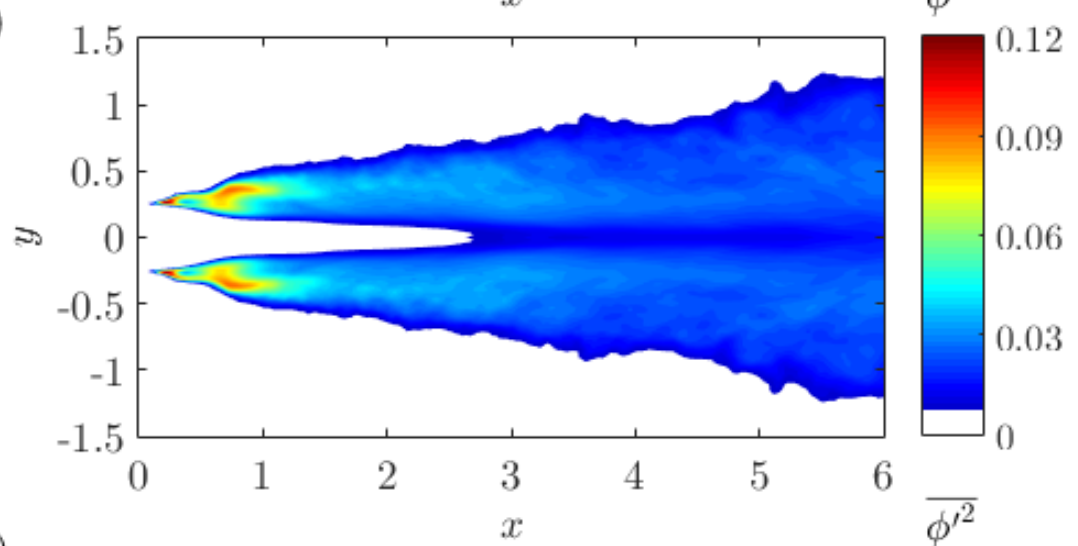

(d)

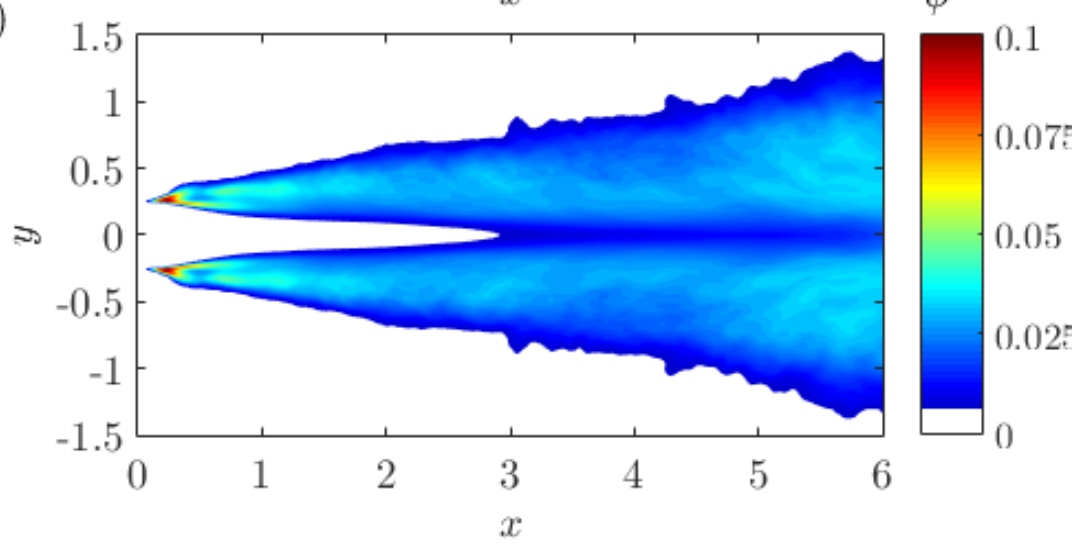

Fig. 3: Fields of the mean passive scalar variance. (a) $R e=5,000$; (b) $R e=10,000$; (c) $R e=20,000$; (d) $R e=40,000$. 
(a)

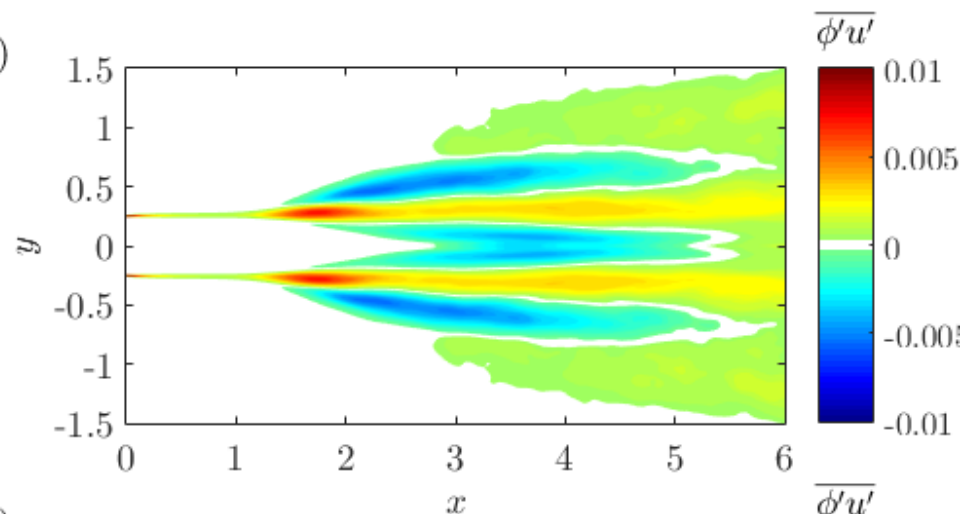

(b)

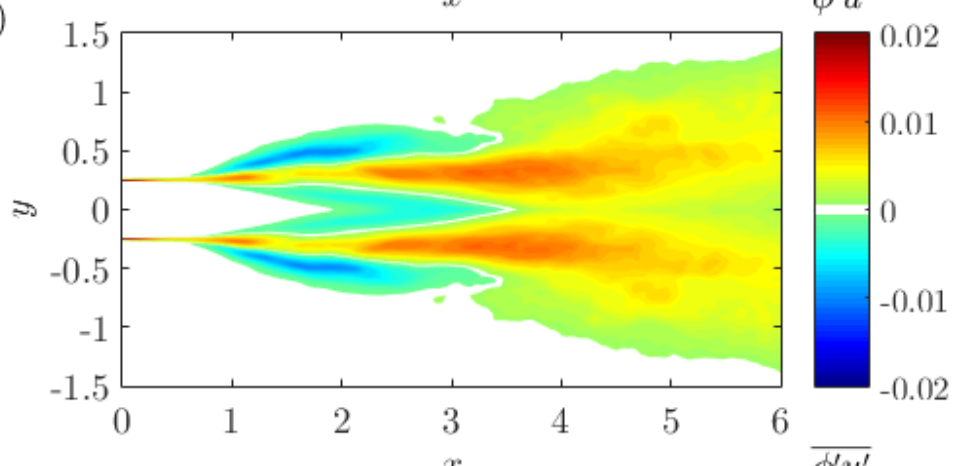

(c)

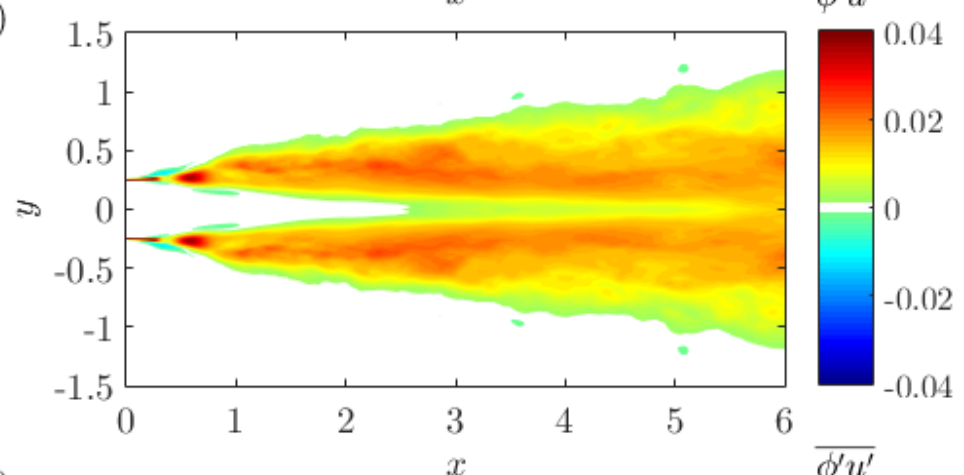

(d)

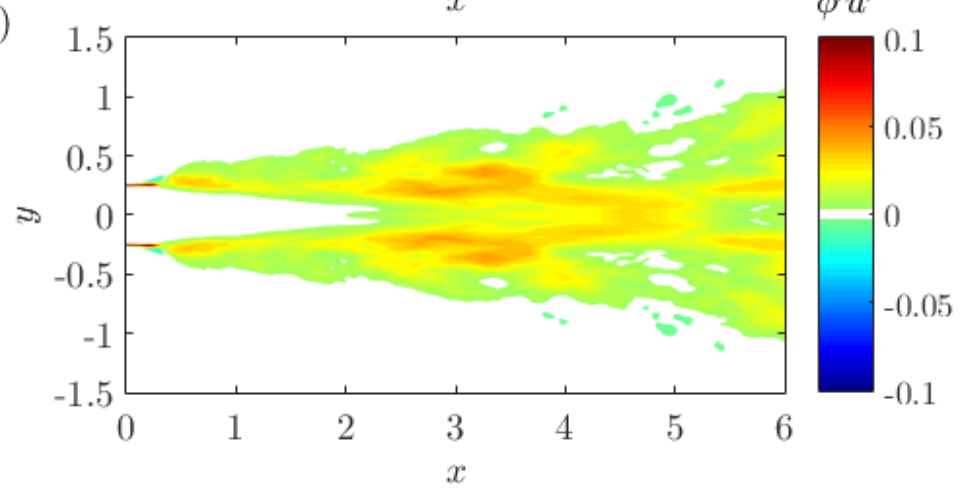

Fig. 4: Fields of the axial mean turbulent passive scalar flux. (a) $\operatorname{Re}=5,000$; (b) $R e=10,000$; (c) $R e=20,000$; (d) $\operatorname{Re}=40,000$. 
(a)
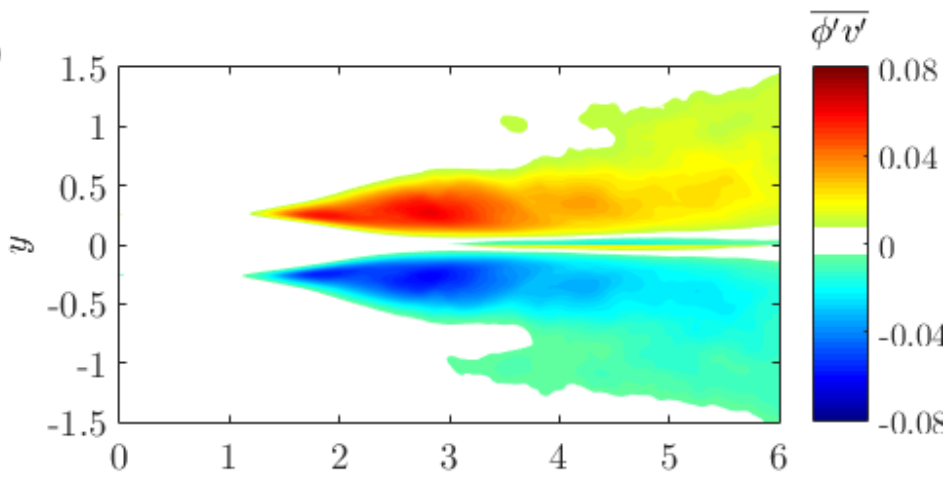

(b)
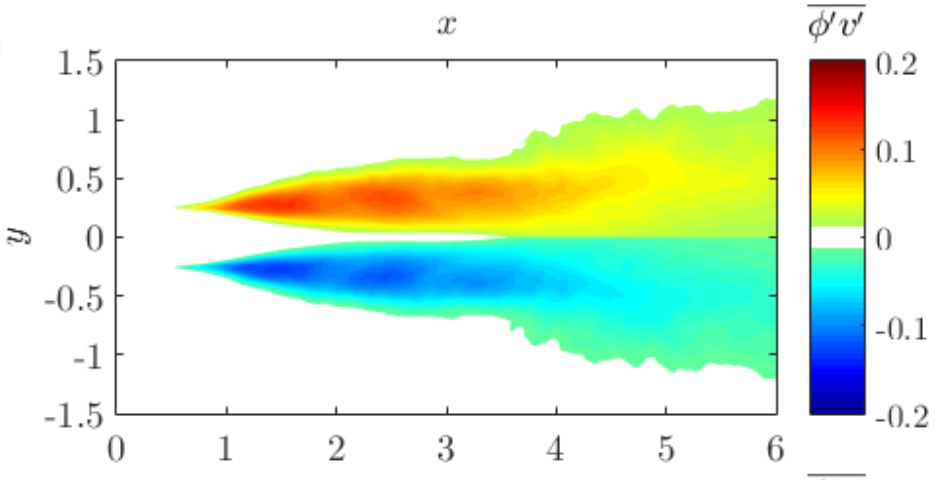

(c)

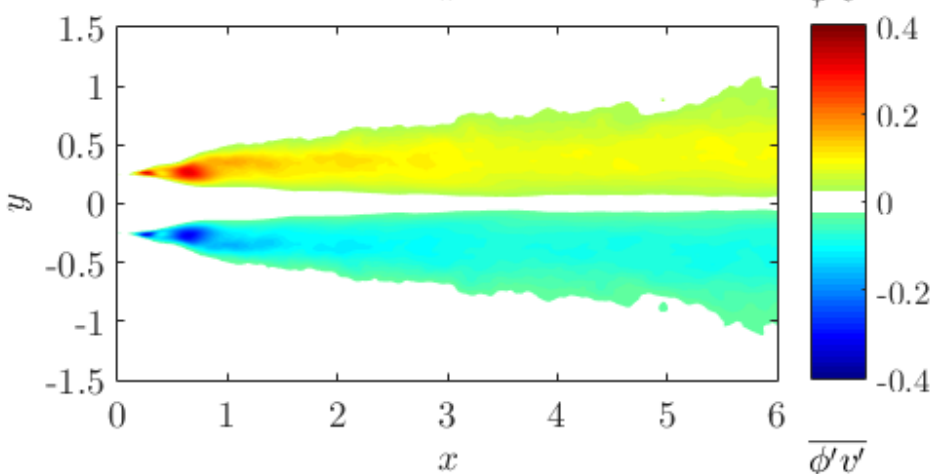

(d)

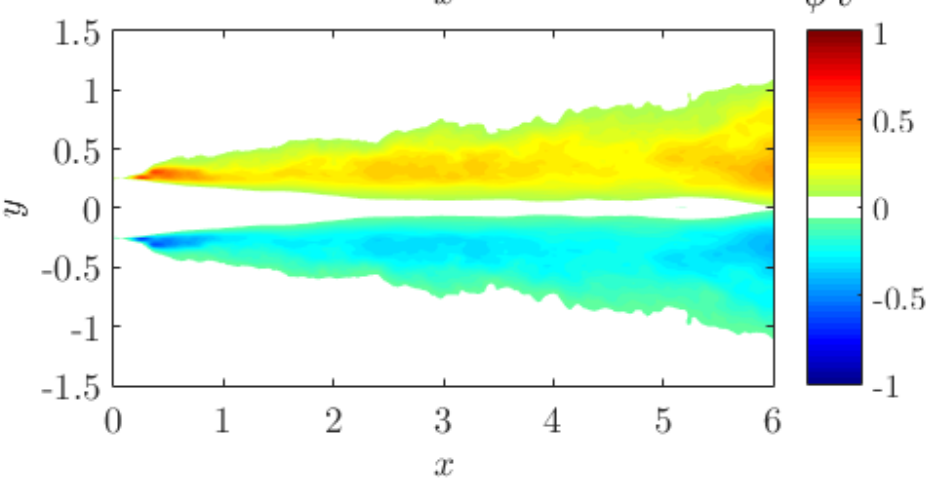

Fig. 5: Fields of the transversal mean turbulent passive scalar flux. (a) $\operatorname{Re}=5,000$; (b) $R e=10,000$; (c) $R e=20,000$; (d) $R e=40,000$. 
(a)

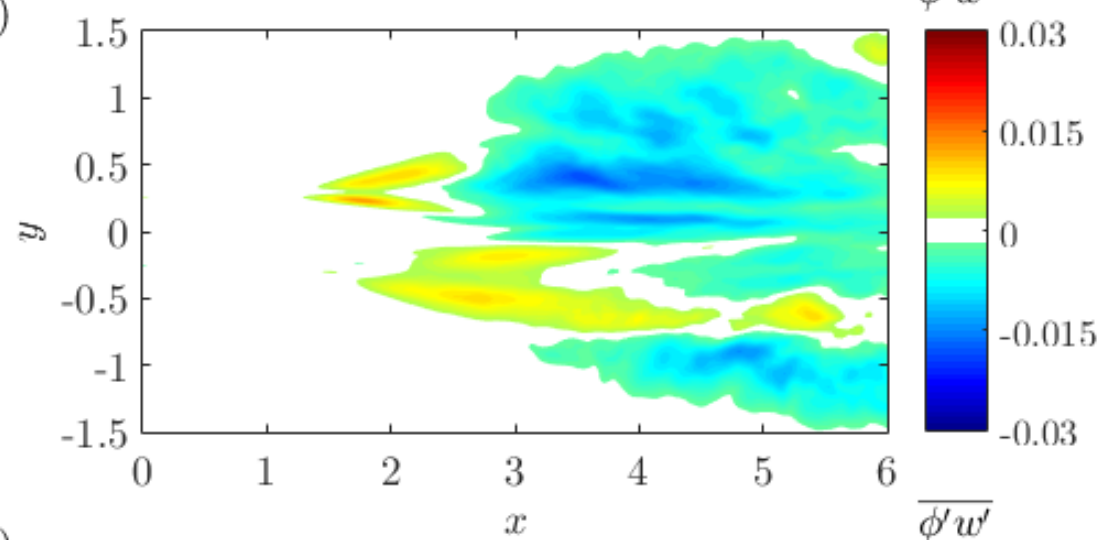

(b)

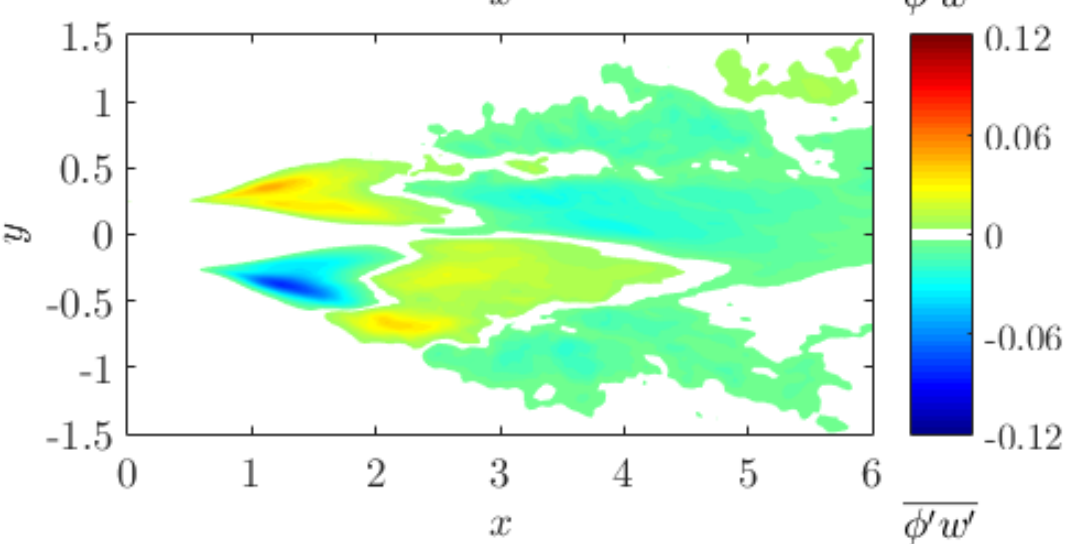

(c)

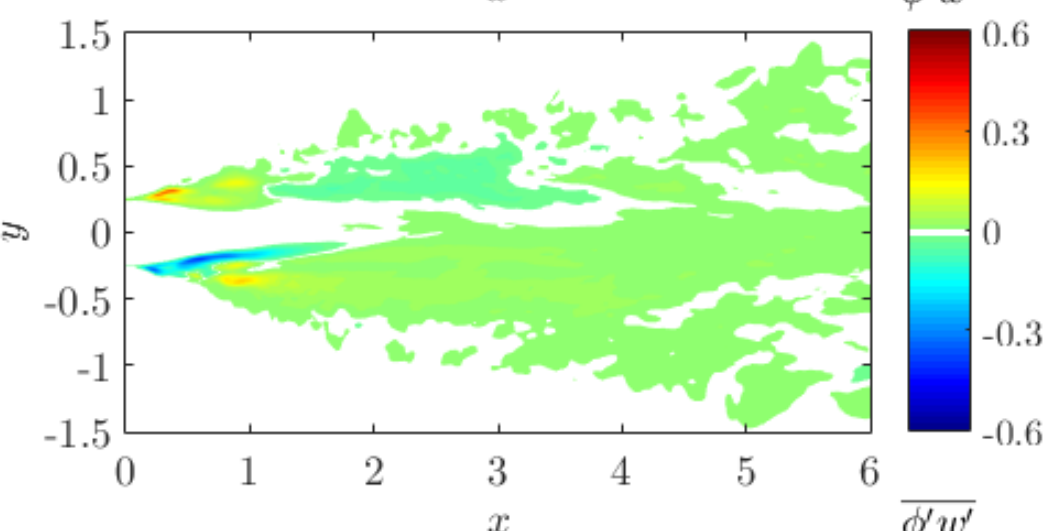

(d)

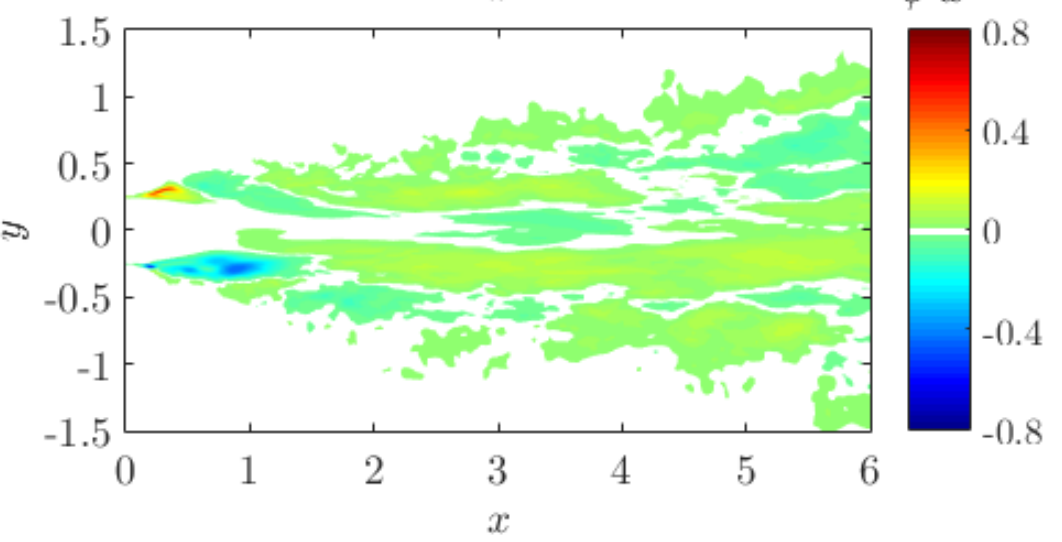

Fig. 6: Fields of the z-direction mean turbulent passive scalar flux. (a) $\operatorname{Re}=5,000$; (b) $\operatorname{Re}=10,000$; (c) $\operatorname{Re}=20,000$; (d) $R e=40,000$. 
(a)
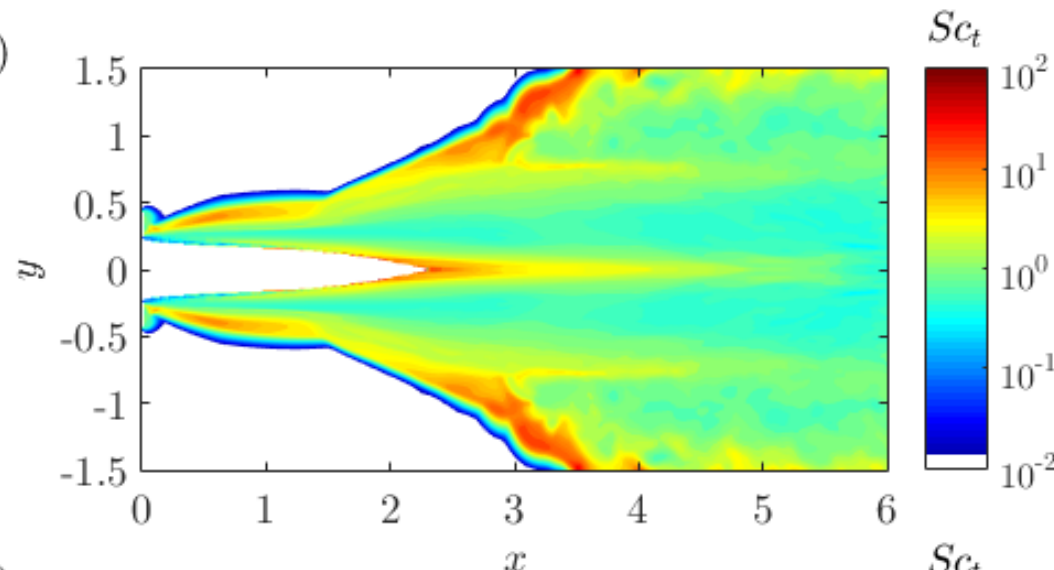

(b)

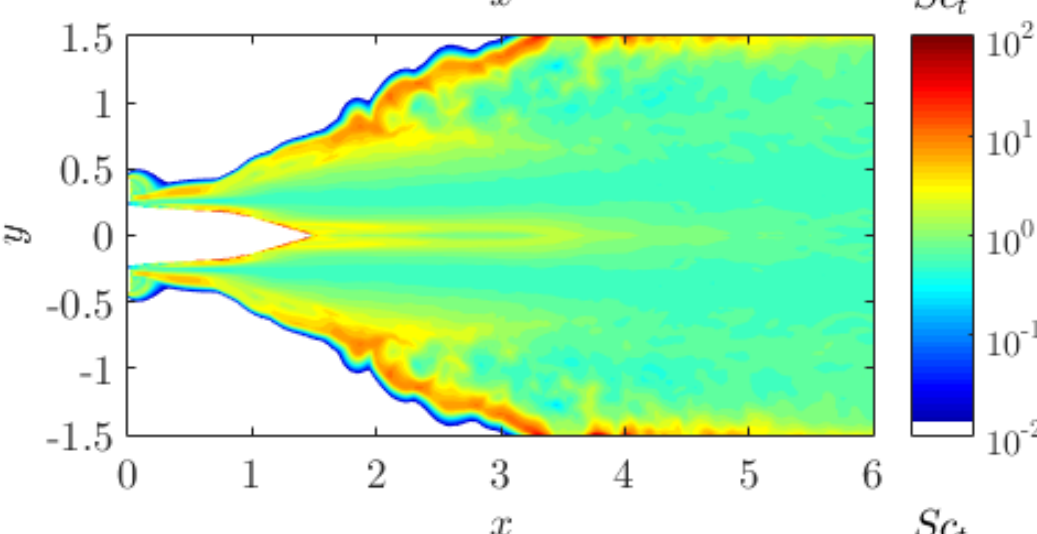

(c)
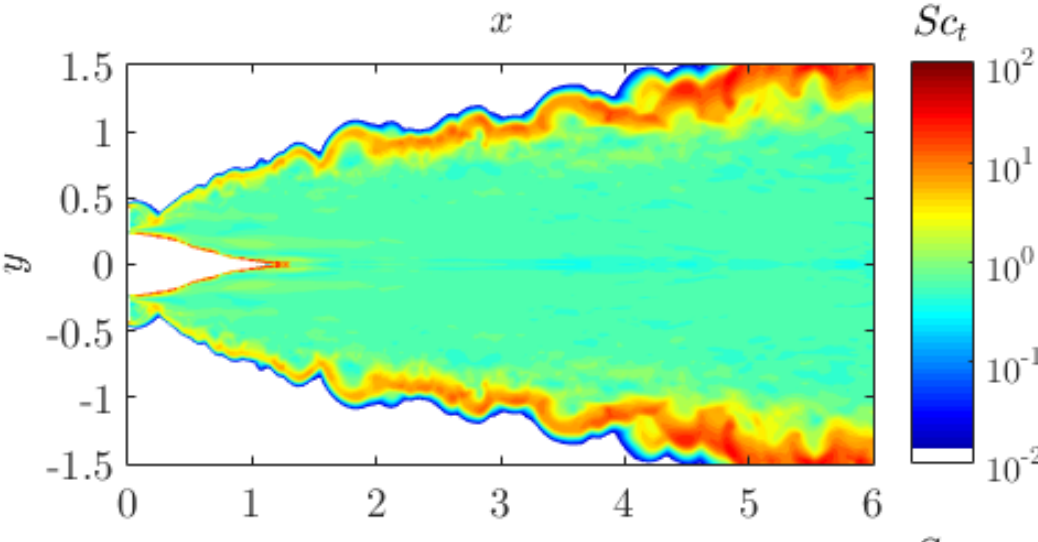

(d)

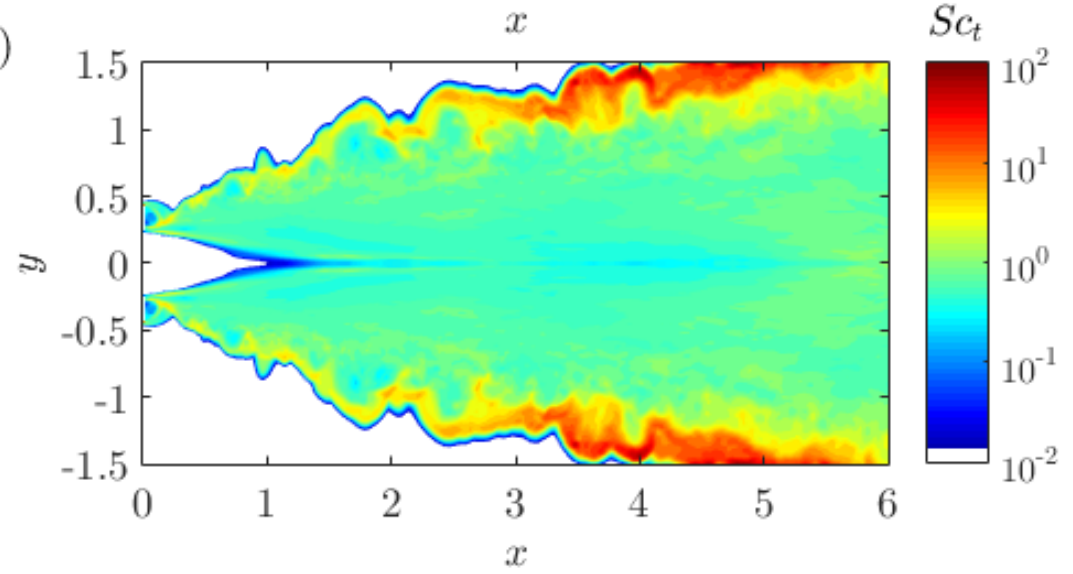

Fig. 7: Turbulent Prandtl or Schmidt number. (a) $R e=5,000 ;$ (b) $R e=10,000 ;$ (c) $R e=20,000 ;$ (d) $R e=40,000$. 
(a)

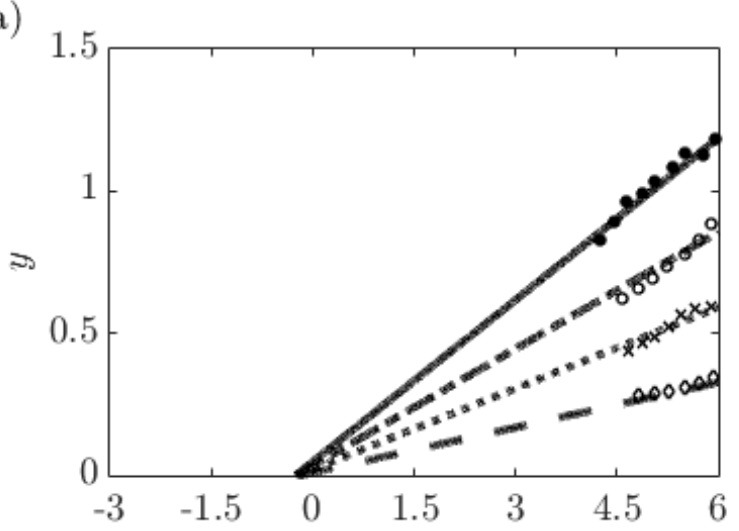

(c)

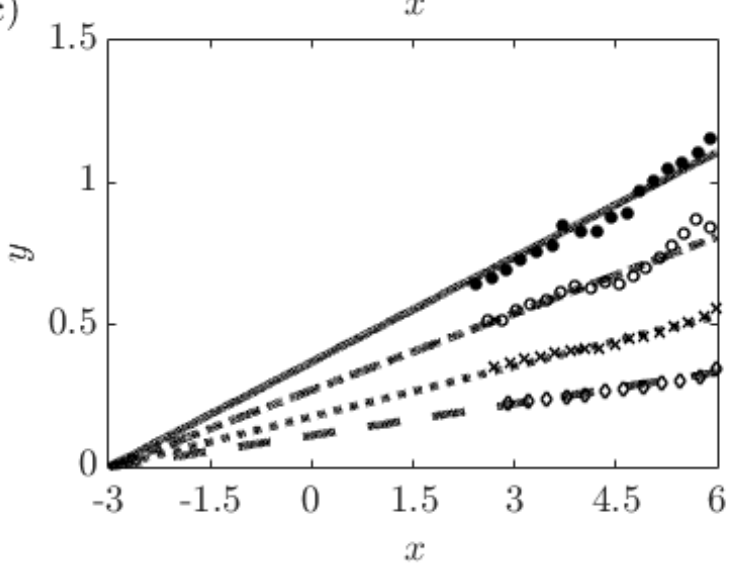

(b)

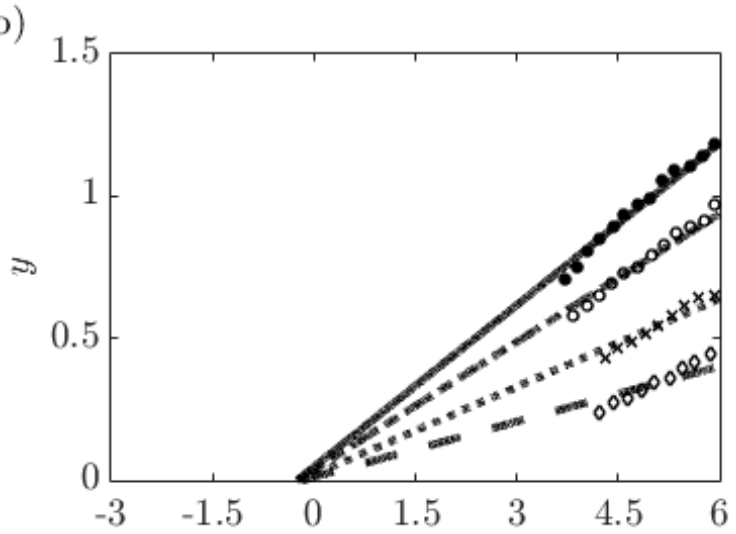

(d)

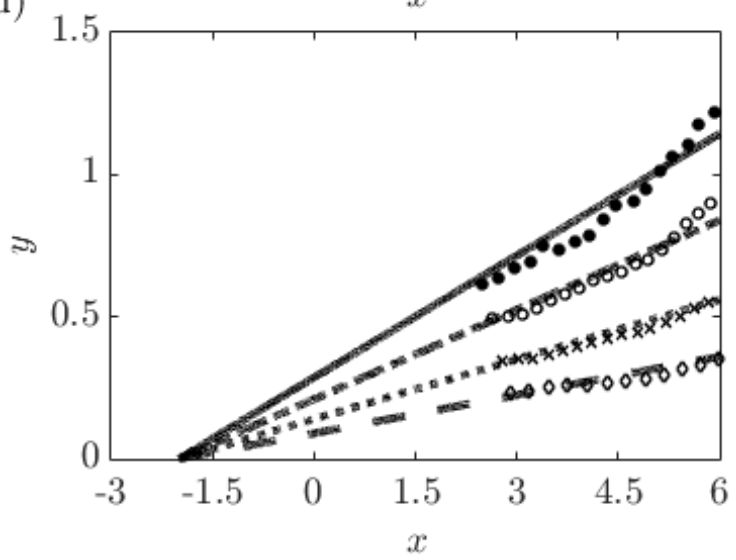

Fig. 8: Mean passive scalar iso-curves in FDR. $\Phi=0.1$, data (๑), regression (-). $\Phi=0.4$, data (०), regression (-.). $\Phi=0.7$, data (x), regression (....). $\Phi=0.9$, data ( () , regression (--). (a) $\operatorname{Re}=5000 ;$ (b) $\operatorname{Re}=10,000 ;$ (c) $\operatorname{Re}=$ 20,000; (d) $\operatorname{Re}=40,000$. 

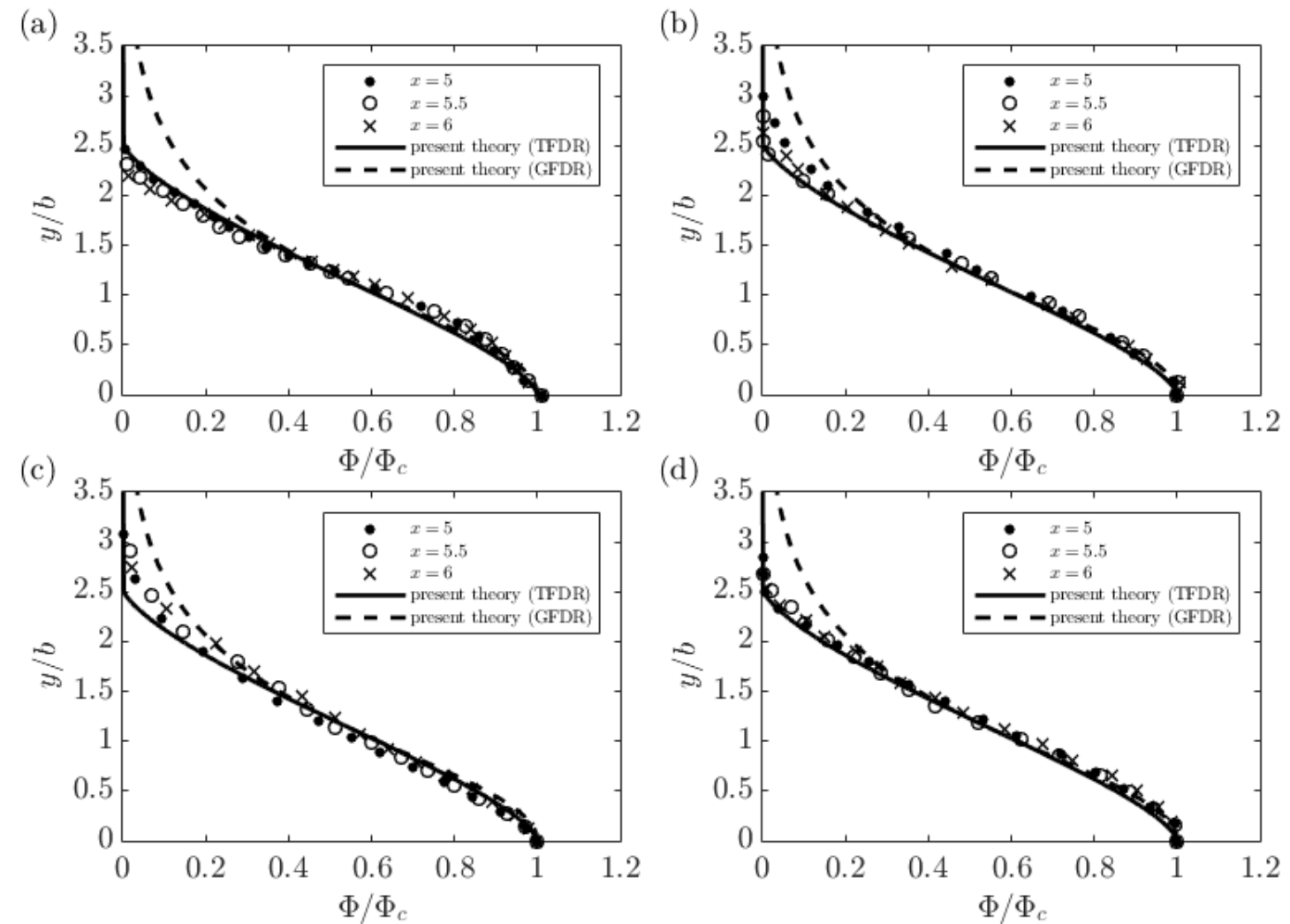

Fig. 9: Mean passive-scalar profile in the FDR. Numerical and "Tollmien-like for the FDR" (TFDR) and "Görtler like for the FDR" (GFDR) theoretical results. (a) $R e=5,000$; (b) $R e=10,000$; (c) $R e=20,000$; (d) $R e=40,000$. 